\title{
ADIABATIC ENSEMBLE CONTROL OF A CONTINUUM OF QUANTUM SYSTEMS*
}

\author{
NICOLAS AUGIER $\dagger^{\dagger}$ UGO BOSCAIN ${ }^{+}$, MARIO SIGALOTTI ${ }^{8 \uparrow}$
}

\begin{abstract}
In this article we discuss how to control a parameter-dependent family of quantum systems. Our technique is based on adiabatic approximation theory and on the presence of curves of conical eigenvalue intersections of the controlled Hamiltonian. As particular cases, we recover chirped pulses for two-level quantum systems and counter-intuitive solutions for three-level stimulated Raman adiabatic passage (STIRAP). The proposed technique works for systems evolving both in finitedimensional and infinite-dimensional Hilbert spaces. We show that the assumptions guaranteeing ensemble controllability are structurally stable with respect to perturbations of the parametrized family of systems.
\end{abstract}

1. Introduction. The problem of controlling quantum mechanical systems has undergone a huge development in recent years, motivated by its applications in manipulation of single spins, photons or atoms, optical spectroscopy, photochemistry, nuclear magnetic resonance, and quantum information processing (see, for instance, $[4,8,13,19,21,24]$ and references therein).

In this paper we are concerned with the problem of ensemble controllability, namely the problem of controlling using finitely many scalar controls (typically few of them, e.g., two or three) a family of systems having slightly different parameters. Equivalently, the problem is to control a quantum system for which certain parameters are unknown. Such a problem arises very often in practical applications. For instance, the dynamics of different spins in a nuclear magnetic resonance sample are characterized by different sets of parameters.

Notice that in such a problem the assumption that the unknown parameter belongs to a continuous set plays an important role. If the parameter belonged to a discrete set, then the classical techniques of controllability could be applied to the system written in a higher-dimensional space (see, for instance, $[6,20]$ ).

The ensemble controllability problem is studied in quantum mechanics since a long time. In particular, it is known that the so-called chirped pulses for two-level systems have good robustness properties with respect to parameter uncertainties [14, 40]. Similarly, the so-called counterintuitive pulses for STIRAP processes in three-level quantum systems are known to realize a population transfer even if certain parameters are unknown $[22,39,41]$. Ensemble controllability is often tackled in experimental situations via direct optimization methods (see, for instance, $[15,42]$ ). The mathematical aspects of the ensemble controllability problem have been addressed, in particular, in $[5,17,33,34]$ for a spin- $\frac{1}{2}$ system whose Larmor frequency is not known precisely. In [31] the effect of chirped pulses for a class of many-level systems is analyzed using adiabatic techniques.

In this paper we present a technique based on adiabatic approximation and on the presence of conical intersections between eigenvalues (see Figure 2.1). We are mainly considering the situation in which the unknown parameters vary in a one-

*A PRELIMINARY VERSION OF THIS PAPER, CONTAINING PARTIAL RESULTS, HAS BEEN PUBLISHED AS A CONFERENCE PROCEEDING IN [12]

${ }^{\dagger}$ CMAP, ÉCOLE POLYTECHNIQUE, UNIVERSITÉ PARIS-SACLAY

$\ddagger$ CNRS

§INRIA

`SORBONNE UNIVERSITÉ, UNIVERSITÉ PARIS-DIDEROT SPC, CNRS, INRIA, LABORATOIRE JACQUES-LOUIS LIONS 
dimensional set (although in several practical applications, as in the use of chirped pulses or in the STIRAP process, we admit the unknown parameters to vary in higherdimensional spaces). We recall that eigenvalue intersections are generically conical for finite-dimensional systems having a real-symmetric (respectively, Hermitian) Hamiltonian in presence of 2 (respectively, 3) controls and that such a genericity property also extends to systems evolving in infinite-dimensional spaces (see Section 6 for more details).

Our technique is based on the simple idea that if two levels of a quantum system are connected by a conical intersection, then generically a one-parameter perturbation results in a one-dimensional manifold of conical intersections, each one corresponding to a value of the parameter. Then an adiabatic path in the space of controls that passes through such a one-dimensional manifold realizes a transfer for all systems (see Figure 2.4).

It is interesting to notice that such an idea explains and generalizes what happens for chirped pulses. The fact that chirped pulses exploit the presence of curves of conical intersections was not noticed before, because of the special symmetries of the system (see Section 4).

Our main result can be roughly resumed in a statement like this: assume that for a given parameter the spectrum of the systems is conically connected (i.e., every energy level is connected to the adjacent ones by conical eigenvalue intersections). Assume that by modifying the parameter the eigenvalue intersections remain conical and describe disjoint 1-d manifolds in the set of admissible controls. Assume, in addition, that the space of controls minus the support of such curves is pathwise connected. Then the system is approximately ensemble controllable. Such a result proves the robustness of adiabatic transitions with respect to variations of the parameters of the system. We recall that the conically connectedness hypothesis implies the exact controllability of each individual system [10].

Our result permits to obtain only transitions between eigenstates of the controlled Hamiltonian, and hence it is less general than the one in [5] (where initial and final conditions can depend on the parameter). However, it can be applied to a much more general class of systems (in particular, it is not restricted to two-level systems). Actually, our result permits to establish a little more than controllability between eigenstates, namely it states that it is possible to induce any arbitrary permutation within a basis of eigenstates of the controlled Hamiltonian. In this sense it generalizes [31], where a result of this kind was presented for a specific class of systems. As it always happens when using adiabatic theory to prove controllability properties, the result is constructive in the sense that it provides a simple algorithmic procedure for obtaining the required control laws.

It should be noticed that the technique that we present here is the only one that applies to the ensemble control of quantum systems evolving in an infinite-dimensional Hilbert space.

The key tool on which we base our reasoning is a uniform adiabatic theorem for systems having conical eigenvalues intersections. A crucial role is played by a technical result guaranteeing that a Hamiltonian that depends $\mathcal{C}^{k}$ on a parameter and whose eigenvalues, seen as functions of such a parameter, are conical, admits an orthonormal family of eigenstates depending $\mathcal{C}^{k-1}$ on the parameter. The regularity of eigenvalues and eigenvectors is well established in the analytic setting [28] and has been studied in the $\mathcal{C}^{\infty}$ case [23]. We prefer to work in the $\mathcal{C}^{k}$ setting because of its generality and since the analytic setting is less natural when studying structural stability and genericity of the assumptions on eigenvalue intersections. Our result is 
in the same spirit as a recent result on the regularity of projectors obtained in [18], of which we became aware while preparing the final version of this article. The regularity of eigenpairs is a useful complement to the classical adiabatic theory in presence of conical intersections, where it is usually assumed that eigenpairs are $\mathcal{C}^{2}$ with respect to the parameter. With our result one can replace such a regularity assumption with the more easily verifiable hypothesis on the $\mathcal{C}^{3}$ regularity of the Hamiltonian itself.

It should be mentioned that the ensemble controllability problem is not restricted to quantum systems. In particular, in $[26,32,35]$ such a problem has been addressed for linear systems. The case of driftless nonlinear systems has been studied in [3], thanks to an infinite-dimensional version of the Rashevsky-Chow theorem.

The paper is organized as follows: In Section 2 we set the ensemble controllability problem and we state our sufficient conditions for approximate ensemble controllability in finite-dimensional spaces. The proof of the sufficiency of such conditions in given in Section 3. The proof of the technical result on the regularity of eigenpairs is postponed to the appendix. In Section 4 we apply the general result to the case of two-level systems, recovering the classical results on chirped pulses. The extension to permutations within a basis of eigenvectors is studied in Section 5. The genericity of the conditions appearing in the sufficient conditions for approximate ensemble controllability is discussed in Section 6. In Section 7 we consider the case in which the Hamiltonian depends on more than one parameter and we illustrate our results on the STIRAP process. Finally, in Section 8, we discuss the extensions to the case of infinite-dimensional spaces, presenting as an example a version of the classical Eberly-Law model.

2. Basic definitions and statement of the main results in the finitedimensional case. For every $n \in N$, let $\llbracket 1, n \rrbracket$ denote the set of integers $\{1, \ldots, n\}$. Let $\mathbf{U}$ be an open connected subset of $\mathbb{R}^{d}, d \geq 2$.

We consider the controlled Schrödinger equation in $\mathbb{C}^{N}, N \in \mathbb{N}$,

$$
i \dot{\psi}=H^{\alpha}(u(t)) \psi
$$

where $u: \mathbb{R} \rightarrow \mathbf{U}$ is a $L^{\infty}$ map. Here $\alpha$ is a time-independent parameter belonging to a compact interval $\left[\alpha_{0}, \alpha_{1}\right] \subset \mathbb{R}$. Each matrix $H^{\alpha}(u)$ belongs to the set $\operatorname{Herm}(N)$ of $N \times N$ Hermitian matrices. The map $(\alpha, u) \mapsto H^{\alpha}(u)$ is sufficiently regular, as it will be specified later on.

Denote the spectrum of $H^{\alpha}(u)$ by $\left(\lambda_{j}^{\alpha}(u)\right)_{j=1}^{N}$ where $j \mapsto \lambda_{j}^{\alpha}(u)$ is the nondecreasing sequence of eigenvalues of $H^{\alpha}(u)$ repeated according to their multiplicities. We also write $\left(\phi_{1}^{\alpha}(u), \ldots, \phi_{N}^{\alpha}(u)\right)$ to denote an orthonormal basis of associated eigenvectors.

DeFINITION 2.1. We say that system (2.1) is ensemble approximately controllable between eigenstates if for every $\varepsilon>0, j, k \in \llbracket 1, N \rrbracket$ and $u_{0}, u_{1} \in \mathbf{U}$ such that $\lambda_{j}^{\alpha}\left(u_{0}\right)$ and $\lambda_{k}^{\alpha}\left(u_{1}\right)$ are simple for every $\alpha \in\left[\alpha_{0}, \alpha_{1}\right]$, there exists a control $u(\cdot):[0, T] \rightarrow \mathbf{U}$ such that for every $\alpha \in\left[\alpha_{0}, \alpha_{1}\right]$ the solution of (2.1) with initial condition $\psi^{\alpha}(0)=\phi_{j}^{\alpha}\left(u_{0}\right)$ satisfies $\left\|\psi^{\alpha}(T)-e^{i \theta} \phi_{k}^{\alpha}\left(u_{1}\right)\right\|<\varepsilon$ for some $\theta \in \mathbb{R}$ (possibly depending on $\alpha$ and $\varepsilon$ ).

REMARK 2.2. The typical case of interest is when $u=0 \in \mathbf{U}$ represents an isolated (i.e., uncontrolled) system (the so-called drift Hamiltonian) and one seeks to steer $\phi_{j}^{\alpha}(0)$ towards $\phi_{k}^{\alpha}(0)$ for some $j, k \in \llbracket 1, N \rrbracket$.

Definition 2.3. Let us fix $\alpha \in\left[\alpha_{0}, \alpha_{1}\right]$. We say that $\bar{u} \in \mathbf{U}$ is a conical intersection between $\lambda_{j}^{\alpha}$ and $\lambda_{j+1}^{\alpha}$ if $\lambda_{j-1}^{\alpha}(\bar{u})<\lambda_{j}^{\alpha}(\bar{u})=\lambda_{j+1}^{\alpha}(\bar{u})<\lambda_{j+2}^{\alpha}(\bar{u})$ and there exists 
$c>0$ such that for every $v$ in a neighborhood of $\bar{u}$ in $\mathbf{U}$, we have

$$
\left\|\lambda_{j}^{\alpha}(v)-\lambda_{j+1}^{\alpha}(v)\right\| \geq c\|\bar{u}-v\| .
$$

(As example of conical intersection is shown in Figure 2.1.)

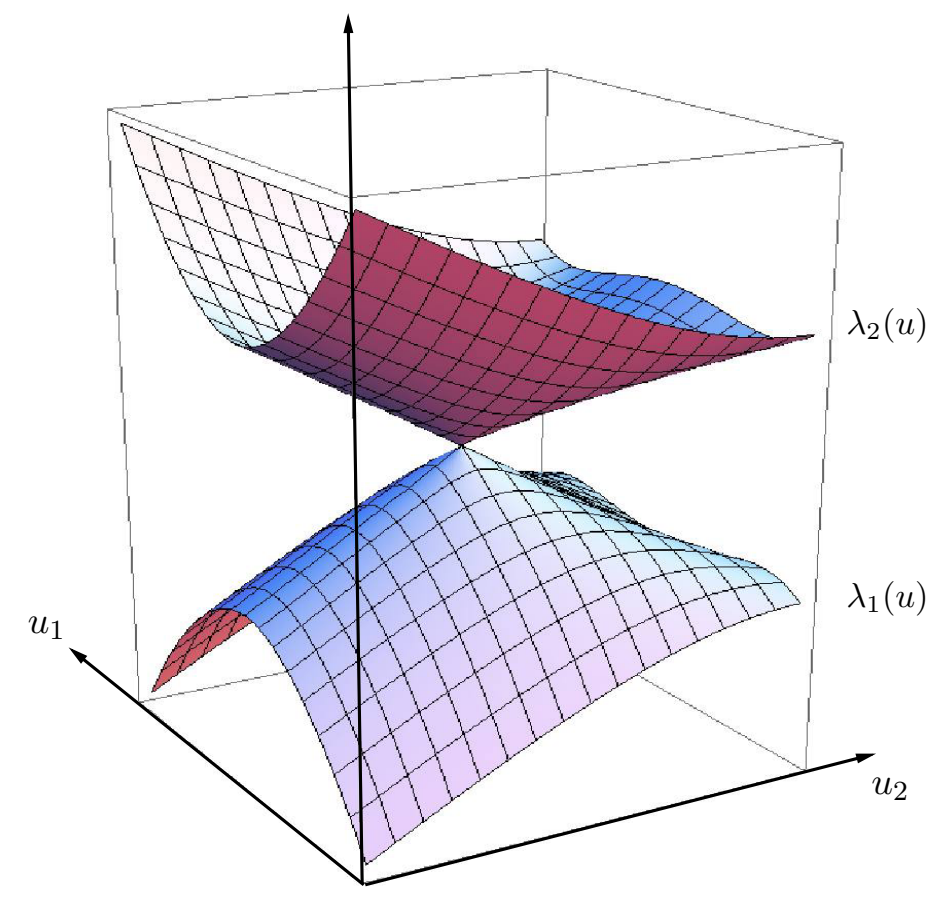

FIG. 2.1. An example of conical intersection in the case $d=N=2$.

Let us denote by $\gamma_{j}$ the projection of the set $\left\{(u, \alpha) \in \mathbf{U} \times\left[\alpha_{0}, \alpha_{1}\right] \mid \lambda_{j}^{\alpha}(u)=\right.$ $\left.\lambda_{j+1}^{\alpha}(u)\right\}$ onto the $u$-component, that is,

$$
\begin{aligned}
\gamma_{0} & =\emptyset, \\
\gamma_{j} & =\left\{u \in \mathbf{U} \mid \exists \alpha \in\left[\alpha_{0}, \alpha_{1}\right] \text { such that } \lambda_{j}^{\alpha}(u)=\lambda_{j+1}^{\alpha}(u)\right\}, \quad j \in \llbracket 1, N-1 \rrbracket, \\
\gamma_{N} & =\emptyset .
\end{aligned}
$$

Assumption $A_{j}$. There exist a connected component $\hat{\gamma}_{j}$ of $\gamma_{j}$ and a map $\beta_{j}$ : $\left[\alpha_{0}, \alpha_{1}\right] \rightarrow \mathbf{U}$ such that $\beta_{j}$ is a $\mathcal{C}^{3}$ embedding and

- $\hat{\gamma}_{j}=\beta_{j}\left(\left[\alpha_{0}, \alpha_{1}\right]\right)$

- $\hat{\gamma}_{j}$ is contained in $\mathbf{U} \backslash\left(\gamma_{j-1} \cup \gamma_{j+1}\right)$

- For every $\alpha \in\left[\alpha_{0}, \alpha_{1}\right], \lambda_{j}^{\alpha}$ and $\lambda_{j+1}^{\alpha}$ have a unique intersection on $\hat{\gamma}_{j}$, which is conical and occurs at $\beta_{j}(\alpha)$.

Moreover the set $\mathbf{U} \backslash\left(\gamma_{j-1} \cup \gamma_{j} \cup \gamma_{j+1}\right)$ is pathwise connected.

REMARK 2.4. The assumption that $\beta_{j}$ is an embedding between the set of parameters $\left[\alpha_{0}, \alpha_{1}\right]$ and $\hat{\gamma}_{j}$ is used here for simplicity and can be relaxed. This will be done in the general context of multi-dimensional sets of parameters in Section 7 (see Assumption $A_{j}^{*}$ ).

The structural stability and the genericity of Assumption $A_{j}$ are discussed in Section 6. In the following theorem we show that the curve $\hat{\gamma}_{j}$ appearing in Assumption 


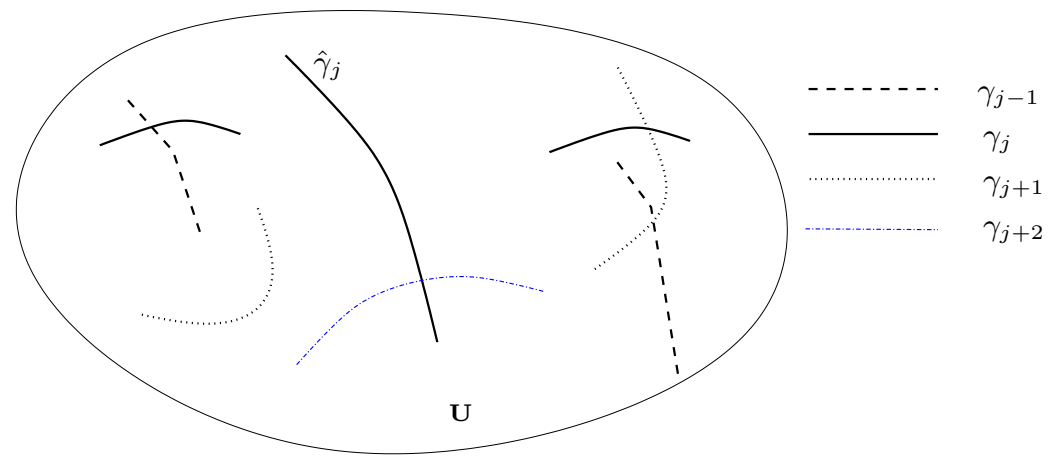

FIG. 2.2. The component $\hat{\gamma}_{j}$ is such that Assumption $A_{j}$ is satisfied

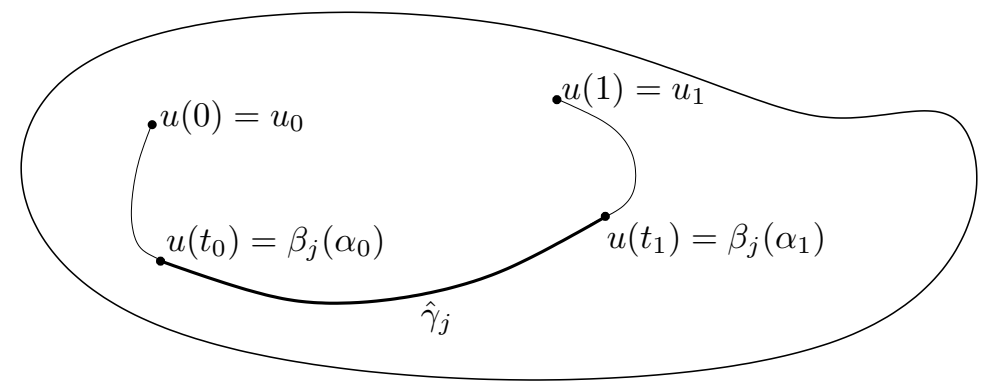

FIG. 2.3. A control $u(\cdot)$ as in the statement of Theorem 2.5.

$A_{j}$ can be used to induce an adiabatic transition between the $j$-th and the $(j+1)$-th eigenstate for every $\alpha$.

Theorem 2.5. Consider a $\mathcal{C}^{3} \operatorname{map}\left[\alpha_{0}, \alpha_{1}\right] \times \mathbf{U} \ni(\alpha, u) \mapsto H^{\alpha}(u) \in \operatorname{Herm}(N)$. Let $j \in \llbracket 1, N-1 \rrbracket$ be such that Assumption $A_{j}$ is satisfied. Take $u_{0}, u_{1} \in \mathbf{U} \backslash\left(\gamma_{j-1} \cup\right.$ $\left.\gamma_{j} \cup \gamma_{j+1}\right)$ and consider a $\mathcal{C}^{3}$ path $u(\cdot):[0,1] \rightarrow \mathbf{U}$ satisfying $u(0)=u_{0}, u(1)=u_{1}$, and such that $\left.u\right|_{\left[t_{0}, t_{1}\right]}$ is a reparameterization of $\beta_{j}$ for some $0<t_{0}<t_{1}<1$ such that $u(t) \notin \gamma_{j-1} \cup \gamma_{j} \cup \gamma_{j+1}$ for every $t \in[0,1] \backslash\left[t_{0}, t_{1}\right]$. (See Figure 2.3.) Assume, moreover, that $\dot{u}(t) \neq 0$ for every $t \in[0,1]$.

Then there exists a constant $C>0$ such that for every $\alpha \in\left[\alpha_{0}, \alpha_{1}\right]$ and $\varepsilon>0$ the solutions $\psi_{\varepsilon}^{\alpha, \pm}$ of

with initial conditions

$$
i \dot{\psi}_{\varepsilon}^{\alpha, \pm}(t)=H^{\alpha}(u(\varepsilon t)) \psi_{\varepsilon}^{\alpha, \pm}(t)
$$

$$
\psi_{\varepsilon}^{\alpha,+}(0)=\phi_{j}^{\alpha}\left(u_{0}\right), \quad \psi_{\varepsilon}^{\alpha,-}(0)=\phi_{j+1}^{\alpha}\left(u_{0}\right)
$$

satisfy

$$
\left\|\psi_{\varepsilon}^{\alpha,+}(1 / \varepsilon)-e^{i \theta^{+}} \phi_{j+1}^{\alpha}\left(u_{1}\right)\right\| \leq C \sqrt{\varepsilon}, \quad\left\|\psi_{\varepsilon}^{\alpha,-}(1 / \varepsilon)-e^{i \theta^{-}} \phi_{j}^{\alpha}\left(u_{1}\right)\right\| \leq C \sqrt{\varepsilon},
$$

for some $\theta^{ \pm} \in \mathbb{R}$.

REMARK 2.6. By unitarity of the evolution, if in the statement of Theorem 2.5 we replace the initial conditions (2.2) by

$$
\left\|\psi_{\varepsilon}^{\alpha,+}(0)-\phi_{j}^{\alpha}\left(u_{0}\right)\right\| \leq c, \quad\left\|\psi_{\varepsilon}^{\alpha,-}(0)-\phi_{j+1}^{\alpha}\left(u_{0}\right)\right\| \leq c
$$




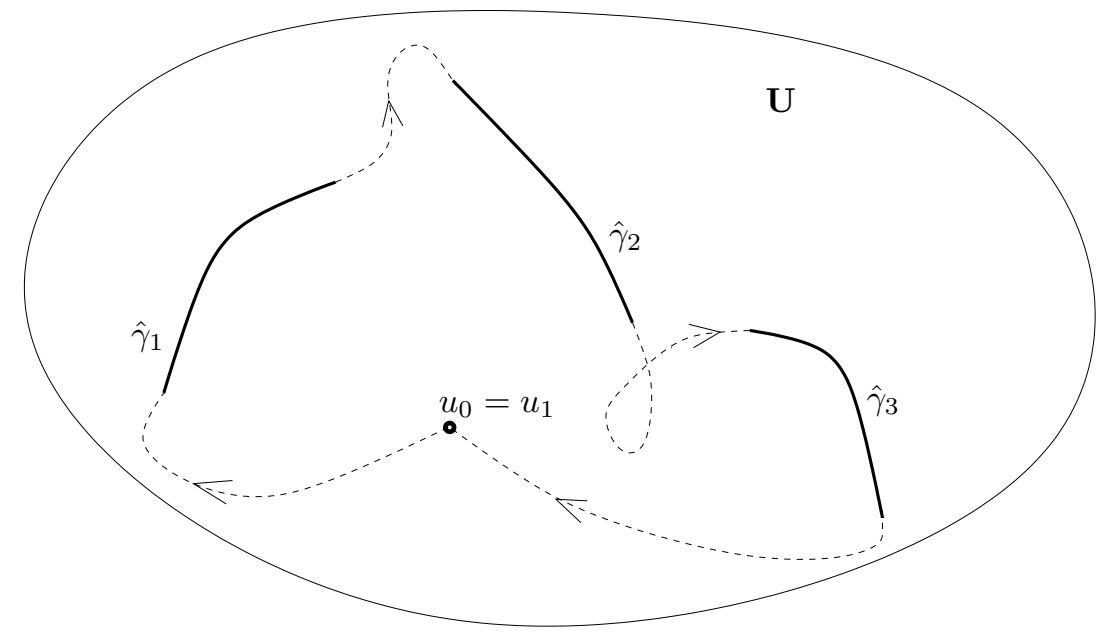

FIG. 2.4. A control realizing an ensemble transition between $\phi_{1}^{\alpha}\left(u_{0}\right)$ and $\phi_{4}^{\alpha}\left(u_{0}\right)$.

then the conclusion (2.3) becomes

$$
\left\|\psi_{\varepsilon}^{\alpha,+}(1 / \varepsilon)-e^{i \theta^{+}} \phi_{j+1}^{\alpha}\left(u_{1}\right)\right\| \leq C \sqrt{\varepsilon}+c, \quad\left\|\psi_{\varepsilon}^{\alpha,-}(1 / \varepsilon)-e^{i \theta^{-}} \phi_{j}^{\alpha}\left(u_{1}\right)\right\| \leq C \sqrt{\varepsilon}+c .
$$

Corollary 2.7. Consider a $\mathcal{C}^{3} \operatorname{map}\left[\alpha_{0}, \alpha_{1}\right] \times \mathbf{U} \ni(\alpha, u) \mapsto H^{\alpha}(u) \in \operatorname{Herm}(N)$. Let Assumption $A_{j}$ be satisfied for every $j \in \llbracket 1, N-1 \rrbracket$. Then (2.1) is ensemble approximately controllable between eigenstates.

The corollary follows from an iterated application of Theorem 2.5 and its proof works by constructing a $\mathcal{C}^{3}$ control such that $u(0)=u_{0}$ and $u(T)=u_{1}$ (where $u_{0}$ and $u_{1}$ are as in Definition 2.1) and going through the curves $\hat{\gamma}_{j}$ as in Figure 2.4. Starting from the second iteration of the application of Theorem 2.5, we use Remark 2.6 with $c$ of order $\sqrt{\varepsilon}$ to guarantee that the adiabatic approximation after each iteration remains of order $\sqrt{\varepsilon}$.

REMARK 2.8. The proof of Theorem 2.5 is based on a uniform adiabatic theorem, which is recalled in next section (Theorem 3.1). Actually, under the additional hypothesis that the curves $\hat{\gamma}_{j}$ are non-mixing for every $\alpha \in\left[\alpha_{0}, \alpha_{1}\right]$, in the sense of $[9$, Section V], one can replace the factor $\sqrt{\varepsilon}$ in (2.3) and (3.1) by $\varepsilon$.

3. Proof of the ensemble controllability result in the finite-dimensional case.

Theorem 3.1. Consider a $\mathcal{C}^{3} \operatorname{map}\left[\alpha_{0}, \alpha_{1}\right] \times \mathbf{U} \ni(\alpha, u) \mapsto H^{\alpha}(u) \in \operatorname{Herm}(N)$. Let $u:[0,1] \rightarrow \mathbf{U}$ be a $\mathcal{C}^{2}$ control. For every $\alpha \in\left[\alpha_{0}, \alpha_{1}\right]$ and $t \in[0,1]$, let $\Lambda_{1}^{\alpha}(t), \ldots, \Lambda_{N}^{\alpha}(t)$ be the eigenvalues of $H^{\alpha}(u(t))$ repeated according to their multiplicities and denote by $\left(\Phi_{1}^{\alpha}(t), \ldots, \Phi_{N}^{\alpha}(t)\right)$ an orthonormal basis of associated eigenvectors.

Assume that for every $\alpha \in\left[\alpha_{0}, \alpha_{1}\right]$ and every $j \in \llbracket 1, N \rrbracket$,

$$
\left(\Lambda_{j}^{\alpha}, \Phi_{j}^{\alpha}\right) \in \mathcal{C}^{2}\left([0,1], \mathbb{R} \times \mathbb{C}^{N}\right) .
$$

Let $j \in \llbracket 1, N-1 \rrbracket$ and assume that for every $\alpha \in\left[\alpha_{0}, \alpha_{1}\right]$, there exists $\tau \in(0,1)$ such that $\Lambda_{j}^{\alpha}(t)$ and $\Lambda_{j+1}^{\alpha}(t)$ are simple for every $t \in[0,1] \backslash\{\tau\}$ and

$$
\Lambda_{j}^{\alpha}(\tau)=\Lambda_{j+1}^{\alpha}(\tau) \neq \Lambda_{m}^{\alpha}(\tau) \text { for } m \neq j, j+1,\left.\quad \frac{d}{d t}\right|_{t=\tau} \Lambda_{j}^{\alpha}(t) \neq\left.\frac{d}{d t}\right|_{t=\tau} \Lambda_{j+1}^{\alpha}(t) .
$$


Then there exists $C>0$ such that for every $\alpha \in\left[\alpha_{0}, \alpha_{1}\right]$, and every $\varepsilon>0$ the solution $\psi=\psi_{\varepsilon}^{\alpha}$ of the equation

$$
i \dot{\psi}(t)=H^{\alpha}(u(\varepsilon t)) \psi(t)
$$

with initial condition $\psi(0)=\Phi_{j}^{\alpha}(0)$ satisfies

$$
\left\|\psi(1 / \varepsilon)-e^{i \theta} \Phi_{j}^{\alpha}(1)\right\| \leq C \sqrt{\varepsilon}
$$

for some $\theta \in \mathbb{R}$.

The proof of this theorem can be obtained by applying the classical adiabatic theorem for every $\alpha$, using the continuity of the corresponding constant $C(\alpha)$ and the compactness of the interval $\left[\alpha_{0}, \alpha_{1}\right]$ (see for instance [27, Theorem 4] and [43, Theorem 1.2]).

In order to guarantee the regularity of eigenvalues and eigenvectors along a regular but not necessarily analytic path in the domain of admissible controls, we are going to apply the following result, whose proof is given in the appendix. (For a result in the same spirit in the infinite-dimensional setting, see [18].)

Lemma 3.2. Let $I$ be an interval in $\mathbb{R}$ and take $H \in \mathcal{C}^{k+1}(I, \operatorname{Herm}(N)), k \geq 0$. For every $t \in I$, let $\lambda_{1}(t) \leq \cdots \leq \lambda_{N}(t)$ be the eigenvalues of $H(t)$, repeated according to their multiplicities. Assume that for every $\bar{t} \in I$ and every $j \in \llbracket 1, N-1 \rrbracket$ such that $\lambda_{j}(\bar{t})=\lambda_{j+1}(\bar{t})$, then $\lambda_{h}(\bar{t}) \neq \lambda_{j}(\bar{t})$ for $h \neq j, j+1$ and there exist $c>0$ and $a$ neighborhood $\bar{I}$ of $\bar{t}$ in I such that

$$
\lambda_{j+1}(t)-\lambda_{j}(t) \geq c|t-\bar{t}|, \quad \text { for every } t \in \bar{I} .
$$

Then there exist $\Lambda_{1}, \ldots, \Lambda_{N} \in \mathcal{C}^{k+1}(I, \mathbb{R})$ and $\Phi_{1}, \ldots, \Phi_{N} \in \mathcal{C}^{k}\left(I, \mathbb{C}^{N}\right)$ such that, for every $t \in I, \Lambda_{1}(t), \ldots, \Lambda_{N}(t)$ are the eigenvalues of $H(t)$ repeated according to their multiplicities and $\left(\Phi_{1}(t), \ldots, \Phi_{N}(t)\right)$ is an orthonormal basis of corresponding eigenvectors. Moreover, $\Phi_{1}, \ldots, \Phi_{n}$ are $\mathcal{C}^{k+1}\left(I, \mathbb{C}^{N}\right)$ if all eigenvalues of $H$ are simple along $I$.

Proof of Theorem 2.5. We are going to apply Theorem 3.1 on the path $u(\cdot)$. Notice that for every $\alpha$ the curve $u(\cdot)$ passes through exactly one point $\bar{u}^{\alpha}$ such that $\lambda_{j}^{\alpha}\left(\bar{u}^{\alpha}\right)=$ $\lambda_{j+1}^{\alpha}\left(\bar{u}^{\alpha}\right)$ and through no point $v \in \mathbf{U}$ where either $\lambda_{j-1}^{\alpha}(v)=\lambda_{j}^{\alpha}(v)$ or $\lambda_{j+1}^{\alpha}(v)=$ $\lambda_{j+2}^{\alpha}(v)$. Denote by $t_{\alpha}$ the time such that $u\left(t_{\alpha}\right)=\bar{u}^{\alpha}$.

By Lemma 3.2 applied to the map $t \mapsto H(u(t))$, we can assume that

$$
[0,1] \ni t \mapsto\left(\Lambda_{j}^{\alpha}(t), \Phi_{j}^{\alpha}(t)\right)= \begin{cases}\left(\lambda_{j}^{\alpha}(u(t)), \phi_{j}^{\alpha}(u(t))\right) & \text { if } t<t_{\alpha} \\ \left(\lambda_{j+1}^{\alpha}(u(t)), \phi_{j+1}^{\alpha}(u(t))\right) & \text { if } t \geq t_{\alpha}\end{cases}
$$

is $\mathcal{C}^{2}$ for every $j \in \llbracket 1, N \rrbracket$ and every $\alpha \in\left[\alpha_{0}, \alpha_{1}\right]$. The application of Theorem 3.1 then concludes the proof of Theorem 2.5.

Remark 3.3. In Theorem 2.5 the control $u(\cdot)$ is assumed to be $\mathcal{C}^{3}$. The $\mathcal{C}^{3}$ regularity could actually be relaxed. The same proof would work, for instance, by assuming $u(\cdot)$ to be continuous, $\mathcal{C}^{3}$ in a neighborhood of $\hat{\gamma}_{j}$ and piecewise $\mathcal{C}^{2}$ elsewhere.

4. Example 1: Two-level system driven by a chirped pulse. A classical example of ensemble control by adiabatic evolution is the famous chirped pulse used in two-level systems. It is used, for instance, for controlling via magnetic fields an ensemble of spin systems with slightly different parameters. We illustrate chirped pulses on the following model

$$
i \frac{d}{d t}\left(\begin{array}{c}
\psi_{1}^{\alpha} \\
\psi_{2}^{\alpha}
\end{array}\right)=\left(\begin{array}{cc}
E+\alpha & \Omega(t) \\
\Omega^{*}(t) & -E-\alpha \\
7 &
\end{array}\right)\left(\begin{array}{c}
\psi_{1}^{\alpha} \\
\psi_{2}^{\alpha}
\end{array}\right),
$$


where $E>0$ is fixed, $\Omega(\cdot) \in L^{\infty}([0, T], \mathbb{C})$ is the control, $\Omega^{*}(t)$ denotes its complex conjugate, and $\alpha$ is a real parameter that can vary in a fixed range $\alpha \in\left[\alpha_{0}, \alpha_{1}\right] \subset$ $(-E, \infty)$. The quantity $E+\alpha$ is called the proper frequency of system (4.1).

Chirped pulses are well studied in the literature (see [14, 40, 31]). We show here below how they naturally show up in the general framework proposed in this paper. Consider the following ensemble approximate controllability problem for (4.1): we want to construct explicitly a control steering, for every value of $\alpha \in\left[\alpha_{0}, \alpha_{1}\right]$, the eigenstate $\phi_{1}^{\alpha}(0)=(1,0)$ to $\phi_{2}^{\alpha}(0)=(0,1)$ of the Hamiltonian in (4.1) corresponding to $\Omega=0$.

Theorem 2.5 and Corollary 2.7 do not directly apply to system (4.1) since the eigenvalues of the Hamiltonian are simple for every value of $\alpha \in\left[\alpha_{0}, \alpha_{1}\right]$ and $\Omega \in \mathbb{C}$.

Hence we perform a suitable time-dependent change of variables (to recast the system in interaction picture). In order to do so, we consider controls having the form

$$
\Omega(t)=u_{1}(t) e^{-i(2 E t+\Delta(t))},
$$

where $u_{1}(\cdot)$ and $\Delta(\cdot)$ are real-valued, and we set

$$
h^{\alpha}(t)=\left(\begin{array}{cc}
E+\alpha & u_{1}(t) e^{-i(2 E t+\Delta(t))} \\
u_{1}(t) e^{i(2 E t+\Delta(t))} & -E-\alpha
\end{array}\right) .
$$

Let us apply the time-dependent unitary change of variable $\psi^{\alpha}(t)=U(t) \Phi^{\alpha}(t)$ with

$$
U(t)=\left(\begin{array}{cc}
e^{-i E t} & 0 \\
0 & e^{i(E t+\Delta(t))}
\end{array}\right)
$$

Notice that the transformation $U(t)$ preserves (up to phases) the two eigenstates $\phi_{1}^{\alpha}(0)=(1,0)$ and $\phi_{2}^{\alpha}(0)=(0,1)$.

Then $\Phi^{\alpha}$ satisfies the equation $i \frac{d}{d t} \Phi^{\alpha}=\mathcal{H}^{\alpha}(t) \Phi^{\alpha}$ with

$$
\mathcal{H}^{\alpha}(t)=U^{-1}(t) h^{\alpha}(t) U(t)-i U^{-1}(t) \frac{d U}{d t}(t)=\left(\begin{array}{cc}
\alpha & u_{1}(t) \\
u_{1}(t) & -\alpha+\frac{d \Delta}{d t}(t)
\end{array}\right) .
$$

Setting $u_{2}(t)=\frac{d \Delta}{d t}(t)$, we are then left to control the family of systems

$$
i \frac{d}{d t}\left(\begin{array}{c}
\Phi_{1}^{\alpha} \\
\Phi_{2}^{\alpha}
\end{array}\right)=\left(\begin{array}{cc}
\alpha & u_{1}(t) \\
u_{1}(t) & -\alpha+u_{2}(t)
\end{array}\right)\left(\begin{array}{c}
\Phi_{1}^{\alpha} \\
\Phi_{2}^{\alpha}
\end{array}\right)
$$

We apply now Theorem 2.5 to system (4.3). To this purpose we have to check that Assumption $A_{1}$ is satisfied. Set

$$
H^{\alpha}\left(u_{1}, u_{2}\right)=\left(\begin{array}{cc}
\alpha & u_{1} \\
u_{1} & -\alpha+u_{2}
\end{array}\right) .
$$

The eigenvalues of $H^{\alpha}\left(u_{1}, u_{2}\right)$ are

$$
\lambda_{1}^{\alpha}\left(u_{1}, u_{2}\right)=\frac{u_{2}-\sqrt{\left(2 \alpha-u_{2}\right)^{2}+4 u_{1}^{2}}}{2}, \quad \lambda_{2}^{\alpha}\left(u_{1}, u_{2}\right)=\frac{u_{2}+\sqrt{\left(2 \alpha-u_{2}\right)^{2}+4 u_{1}^{2}}}{2} .
$$

For any fixed $\alpha \in\left[\alpha_{0}, \alpha_{1}\right], H^{\alpha}$ has a unique eigenvalue intersection, which is conical and takes place at

$$
\left(u_{1}, u_{2}\right)=(0,2 \alpha)
$$




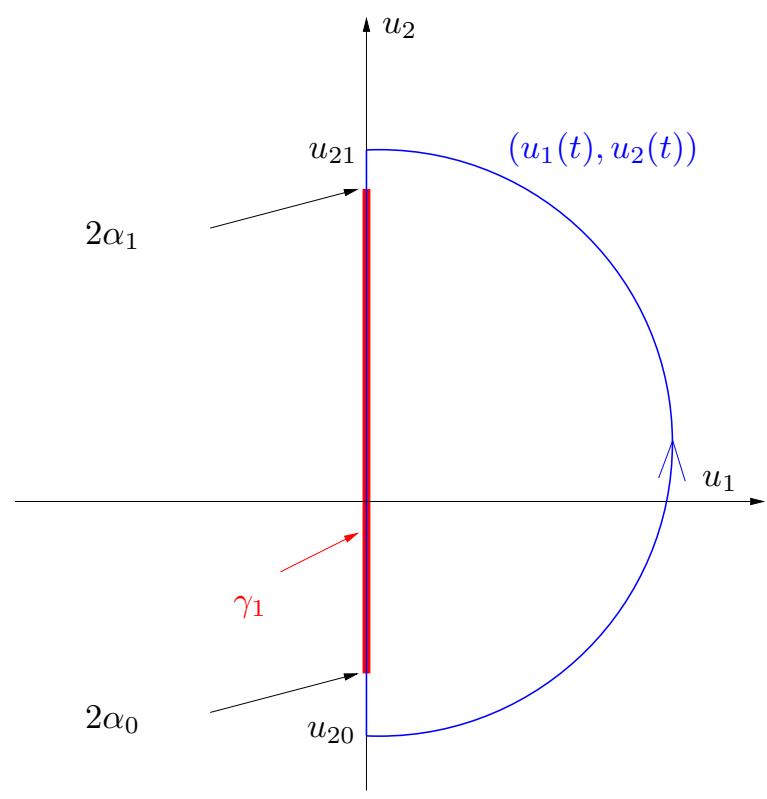

FIG. 4.1. The control $u(\cdot)$.

In other words for each point of the set

$$
\gamma_{1}=\left\{(0,2 \alpha) \in \mathbb{R}^{2} \mid \alpha \in\left[\alpha_{0}, \alpha_{1}\right]\right\}=\{0\} \times\left[2 \alpha_{0}, 2 \alpha_{1}\right]
$$

there is exactly one system having an eigenvalue intersection at such a point, which is, moreover, conical. Assumption $A_{1}$ is then satisfied.

By applying Corollary 2.7 to system (4.3) we deduce the following result.

Proposition 4.1. System (4.3) is ensemble approximately controllable between eigenstates.

The controls used to achieve the controllability stated in Proposition 4.1 can be taken continuous, $\mathcal{C}^{3}$ in a neighborhood of $\gamma_{1}$ and piecewise $\mathcal{C}^{2}$ elsewhere (see Remark 3.3). Notice that the same regularity holds for $\Omega$, according to (4.2).

As a consequence of Proposition 4.1 we can steer (up to phases) system (4.3) (simultaneously with respect to $\alpha \in\left[\alpha_{0}, \alpha_{1}\right]$ ) from $(1,0)$ to an arbitrary neighborhood of $(0,1)$, which are eigenstates of $\mathcal{H}^{\alpha}\left(0, u_{20}\right)$ for every $u_{20} \in \mathbb{R} \backslash\left[2 \alpha_{0}, 2 \alpha_{1}\right]$. Now, since $U(t)$ preserves (up to phases) $(1,0)$ and $(0,1)$, we can conclude that system $(4.1)$ can be steered simultaneously by adiabatic control from $(1,0)$ to $(0,1)$ for every $\alpha \in\left[\alpha_{0}, \alpha_{1}\right]$. Notice that the control $u=\left(0, u_{20}\right)$ corresponds to $\Omega=0$ in the original system, independently of $u_{20}$.

We now construct explicitly the adiabatic control realizing the transition. We start by doing this for (4.3) and then we translate the result for (4.1).

Let $u_{21}$ be in the connected component of $\mathbb{R} \backslash\left[2 \alpha_{0}, 2 \alpha_{1}\right]$ not containing $u_{20}$ (see Figure 4.1). Let $u:[0,1 / 2] \rightarrow \mathbb{R}^{2}$ be a path following a curve contained in the right half-plane connecting $\left(0, u_{20}\right)$ to $\left(0, u_{21}\right)$. Complete the path $u$ by letting $u$ : $[1 / 2,1] \rightarrow \mathbb{R}^{2}$ follow a straight segment from $\left(0, u_{21}\right)$ to $\left(0, u_{20}\right)$. The path $u(\cdot)$ is such that $t \mapsto u(\varepsilon t)$ yields the desired ensemble approximate transition when $\varepsilon \rightarrow 0$.

In terms of the original system, the control $\Omega$ obtained from $u$ as in (4.2) is identically equal to zero on $[1 / 2,1]$. Hence, the evolution corresponding to this portion 

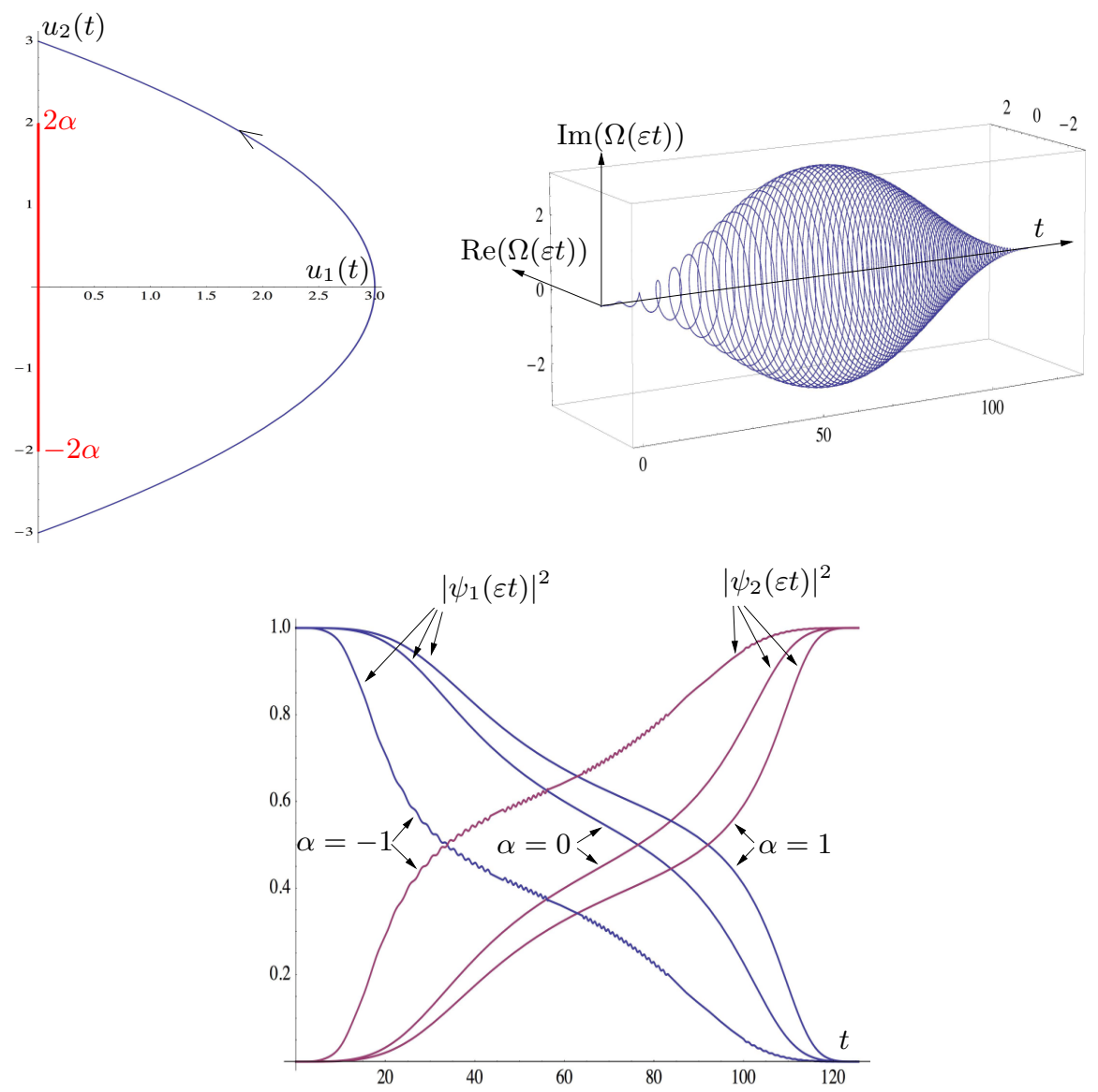

FIG. 4.2. Two-level system driven by a chirped pulse. Here $E=2, \alpha \in[-1,1], u_{1}(t)=$ $\frac{3}{2}(1-\cos (4 \pi t)), u_{2}(t)=-3 \cos (2 \pi t)$, for $t \in\left[0, \frac{1}{2}\right]$ and $\varepsilon=0.004$. The frequency of the pulse is $2 E+u_{2}(\varepsilon t)=4-3 \cos (2 \pi \varepsilon t)$ which varies monotonically between 1 and 7 .

of the control $u$ has no effect on the dynamics and can be ignored. The original system is then controlled by

$$
\Omega(\varepsilon t)=u_{1}(\varepsilon t) e^{-i\left(2 E t+\frac{1}{\varepsilon} \int_{0}^{\varepsilon t} u_{2}(s) d s\right)}
$$

with $t \in\left[0, \frac{1}{2 \varepsilon}\right]$. Notice that $t \mapsto \Omega(\varepsilon t)$ is a complex function with slow-varying modulus and a phase oscillating with a frequency given by

$$
\frac{d}{d t}\left(2 E t+\frac{1}{\varepsilon} \int_{0}^{\varepsilon t} u_{2}(s) d s\right)=2 E+u_{2}(\varepsilon t) .
$$

Since $u_{2}(0)=u_{20}$ and $u_{2}(1 / 2)=u_{21}$, such a frequency slowly varies between two values, one smaller and one larger than every proper frequency $E+\alpha$ of system (4.1) (see Figure 4.2 for an example).

Let us conclude by a remark on the precision of the adiabatic estimate. According to Theorem 2.5, the precision of the transition of system (4.3) corresponding to $u$ : $[0,1] \rightarrow \mathbb{R}^{2}$ is of order $\sqrt{\varepsilon}$. Taking into account, moreover, Remark 2.8 , the precision is of order $\varepsilon$. For what concerns system (4.1), we are just interested, as we have seen, to the restriction of $u$ on the interval $[0,1 / 2]$ and its corresponding control $\Omega$. 


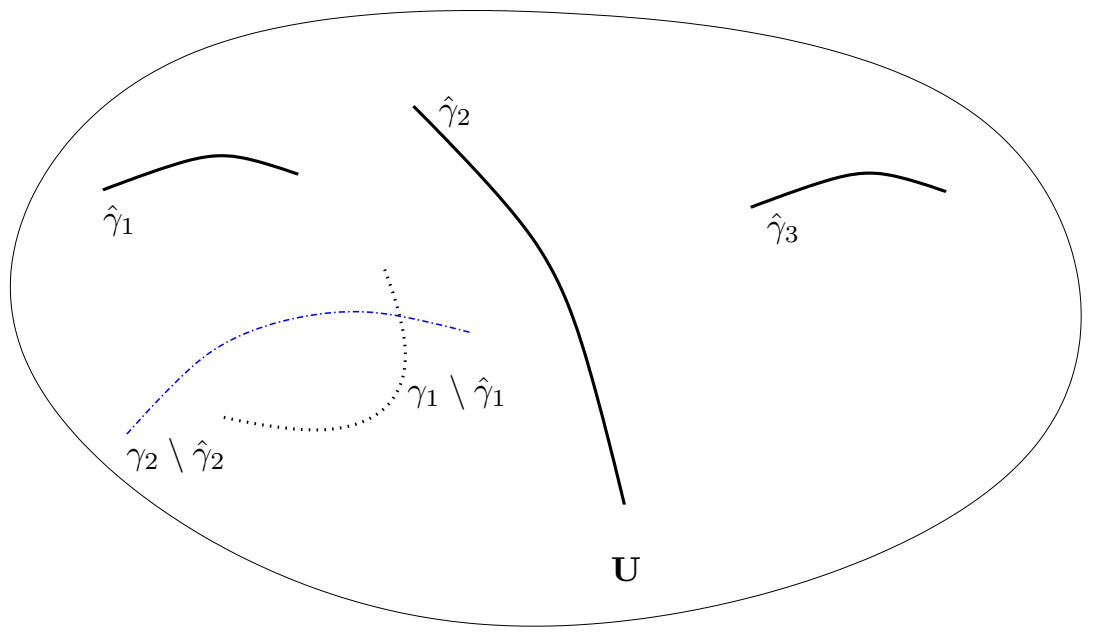

FIG. 5.1. A situation in which Assumption $\mathbf{P}$ is verified.

Hence, since $u$ crosses no eigenvalue intersection on $[0,1 / 2]$, one can conclude that the precision of the adiabatic transition is of order $\varepsilon$ even without recalling Remark 2.8.

5. Permutations. Inspired by [31], we refine in this section our approach in order to select adiabatic controls performing a prescribed permutation of the eigenvectors of the controlled Hamiltonian.

The set of assumptions $A_{j}, j \in \llbracket 1, N-1 \rrbracket$, is replaced by the slightly stronger assumption $\mathbf{P}$ below (see Figure 5.1 for an illustration).

Assumption P. For every $j \in \llbracket 1, N-1 \rrbracket$ there exist a connected component $\hat{\gamma}_{j}$ of $\gamma_{j}$ and a map $\beta_{j}:\left[\alpha_{0}, \alpha_{1}\right] \rightarrow \mathbf{U}$ such that $\beta_{j}$ is a $\mathcal{C}^{3}$ embedding and

- $\hat{\gamma}_{j}=\beta_{j}\left(\left[\alpha_{0}, \alpha_{1}\right]\right)$.

- $\hat{\gamma}_{j}$ is contained in $\mathbf{U} \backslash\left(\cup_{\substack{k \in \llbracket 1, N \rrbracket \\ k \neq j}} \gamma_{k}\right)$

- For every $\alpha \in\left[\alpha_{0}, \alpha_{1}\right], \lambda_{j}^{\alpha}$ and $\lambda_{j+1}^{\alpha}$ have a unique intersection on $\hat{\gamma}_{j}$, which is conical and occurs at $\beta_{j}(\alpha)$.

Moreover the set $\mathbf{U} \backslash\left(\cup_{k \in \llbracket 1, N \rrbracket} \gamma_{k}\right)$ is pathwise connected.

Under Assumption $\mathbf{P}$, Corollary 2.7 can be refined as follows.

TheOrem 5.1. Assume that $\mathbf{P}$ is satisfied. Then for every $u_{0}, u_{1} \in \mathbf{U} \backslash\left(\cup_{k \in \llbracket 1, N \rrbracket} \gamma_{k}\right)$, every $p_{1}, \ldots, p_{N} \in \mathbb{C}$ such that $\left|p_{1}\right|^{2}+\cdots+\left|p_{N}\right|^{2}=1$ and every permutation $\sigma$ : $\llbracket 1, N \rrbracket \rightarrow \llbracket 1, N \rrbracket$, there exists a $\mathcal{C}^{3}$ path $u(\cdot):[0,1] \rightarrow \mathbf{U}$ satisfying $u(0)=u_{0}$ and $u(1)=u_{1}$ and a constant $C>0$ such that for every $\alpha \in\left[\alpha_{0}, \alpha_{1}\right]$ and $\varepsilon>0$ the solution $\psi_{\varepsilon}^{\alpha}$ of

$$
i \dot{\psi}_{\varepsilon}^{\alpha}=H^{\alpha}(u(\varepsilon t)) \psi_{\varepsilon}^{\alpha}
$$

with initial condition $\psi_{\varepsilon}^{\alpha}(0)=\sum_{j=1}^{N} p_{j} \phi_{j}^{\alpha}\left(u_{0}\right)$, satisfies

$$
\left\|\psi_{\varepsilon}^{\alpha}(1 / \varepsilon)-\sum_{j=1}^{N} p_{j} e^{i \theta_{j}} \phi_{\sigma(j)}^{\alpha}\left(u_{1}\right)\right\| \leq C \sqrt{\varepsilon},
$$

for some $\theta_{1}, \ldots, \theta_{N} \in \mathbb{R}$.

Proof. The path $u(\cdot)$ is constructively obtained by requiring it to pass through the curves $\hat{\gamma}_{j}$ in a suitable order. Such an order is identified by the algorithm described below. 

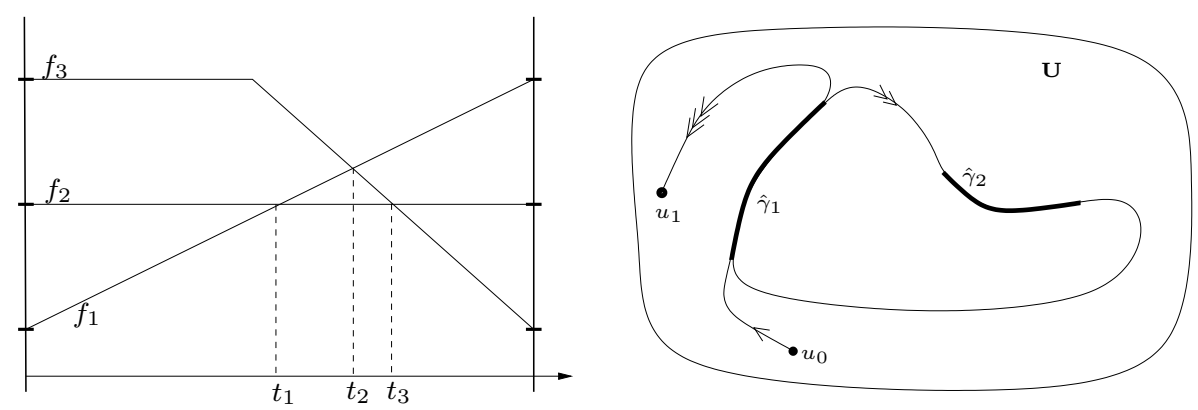

FIG. 5.2. On the left, the functions $f_{j}$ and the times $t_{k}$. On the right, the control $u(\cdot)$. In this case $N=3, \mu=3, \tau(1)=1, \tau(2)=2, \tau(3)=1$. Hence, $u(\cdot)$ passes through $\hat{\gamma}_{1}, \hat{\gamma}_{2}$, and again $\hat{\gamma}_{1}$ while connecting $u_{0}$ to $u_{1}$.

Let $h \in \llbracket 1, N \rrbracket$. We say that the functions $f_{1}, \ldots, f_{h}:[0,1] \rightarrow \mathbb{R}$ satisfy property $\left(\Pi_{h}\right)$ if they are continuous, piecewise affine, such that $f_{j}(0)=j, f_{j}(1)=\sigma(j)$ for $j \in \llbracket 1, h \rrbracket$ and, moreover, if for every $t \in(0,1)$ and $j \neq k, j, k \in \llbracket 1, h \rrbracket$ such that $f_{j}(t)=f_{k}(t)$ we have

- $f_{l}(t) \neq f_{m}(t)$ for every $l \in \llbracket 1, h \rrbracket \backslash\{j, k\}, m \in \llbracket 1, h \rrbracket \backslash\{l\}$,

- $\left(f_{j}(t+\varepsilon)-f_{k}(t+\varepsilon)\right)\left(f_{j}(t-\varepsilon)-f_{k}(t-\varepsilon)\right)<0$ for every $\varepsilon>0$ small enough.

We now construct recursively a set of functions $f_{1}, \ldots, f_{N}$ satisfying $\left(\Pi_{N}\right)$.

Let $f_{1}$ and $f_{2}$ be the affine functions uniquely determined by $f_{1}(0)=1, f_{2}(0)=2$, $f_{1}(1)=\sigma(1), f_{2}(1)=\sigma(2)$. Notice that they satisfy $\left(\Pi_{2}\right)$.

By induction, assume to have selected $f_{1}, \ldots, f_{h}$ satisfying $\left(\Pi_{h}\right)$.

Let $f_{h+1}$ be the affine function satisfying $f_{h+1}(0)=h+1, f_{h+1}(1)=\sigma(h+1)$. If $\left(\Pi_{h+1}\right)$ is satisfied then the recursion step is complete. Otherwise modify $f_{h+1}$ into a continuous function that is constantly equal to $h+1$ in an interval $[0, \eta]$ and affine on $[\eta, 1]$, with the same boundary conditions. For every positive small enough $\eta$ one has that $f_{1}, \ldots, f_{h+1}$ satisfy $\left(\Pi_{h+1}\right)$ and this concludes the induction step.

For every $t \in[0,1]$ let $\sigma_{t}: \llbracket 1, N \rrbracket \rightarrow \llbracket 1, N \rrbracket$ be a permutation such that

$$
f_{\sigma_{t}(1)}(t) \leq f_{\sigma_{t}(2)}(t) \leq \cdots \leq f_{\sigma_{t}(N)}(t) .
$$

Notice that $t \mapsto \sigma_{t}$ is piecewise constant and $\sigma_{0}=\mathrm{Id}, \sigma_{1}=\sigma$.

Let $t_{1}<t_{2}<\cdots<t_{\mu}$ be the values in $(0,1)$ at which the graphs of the functions $f_{1}, \ldots, f_{n}$ intersect (see Figure 5.2). Then $t_{1}, \ldots, t_{\mu}$ are the discontinuity points of $t \mapsto \sigma_{t}$. For every $j \in \llbracket 1, \mu \rrbracket$, let $\tau(j) \in \llbracket 1, N-1 \rrbracket$ be defined by

$$
f_{\sigma_{t_{j}}(\tau(j))}\left(t_{j}\right)=f_{\sigma_{t_{j}}(\tau(j)+1)}\left(t_{j}\right) .
$$

The control $u(\cdot)$ is constructed in such a way that it passes through $\hat{\gamma}_{\tau(1)}, \ldots, \hat{\gamma}_{\tau(\mu)}$. More precisely let $s_{1}, \ldots, s_{\mu}$ be such that $0=: t_{0}<s_{0}<t_{1}<s_{1}<t_{2}<\cdots<$ $s_{\mu-1}<t_{\mu}<s_{\mu}:=1$. Let $u(\cdot):[0,1] \rightarrow \mathbf{U}$ be such that $\dot{u}(t) \neq 0$ for every $t \in[0,1]$, $u(\cdot) \in \mathcal{C}^{3}, u(0)=u_{0}, u(1)=u_{1}$. Moreover for every $j=0, \ldots \mu$, the restriction $\left.u\right|_{\left(t_{j}, s_{j}\right)}$ has values in $\mathbf{U} \backslash\left(\cup_{k \in \llbracket 1, N \rrbracket} \gamma_{k}\right)$ and for $j=1, \ldots \mu$ the curve $\left.u\right|_{\left[s_{j-1}, t_{j}\right]}$ is a reparameterization of $\beta_{\tau(j)}$ (see Figure 5.2).

By construction and by a repeated application of Theorem 2.5 and Remark 2.6, the solution of $i \dot{\psi}_{\varepsilon}^{\alpha}=H^{\alpha}(u(\varepsilon t)) \psi_{\varepsilon}^{\alpha}$ with initial condition $\psi_{\varepsilon}^{\alpha}(0)=\phi_{j}^{\alpha}\left(u_{0}\right)$, satisfies 
for every $t \in \cup_{k=0}^{\mu}\left(t_{k}, s_{k}\right)$

$$
\left\|\psi_{\varepsilon}^{\alpha}(t / \varepsilon)-e^{i \theta_{j}} \phi_{\sigma_{t}(j)}^{\alpha}(u(\varepsilon t))\right\| \leq C \sqrt{\varepsilon},
$$

for some $\theta_{j} \in \mathbb{R}$. The proof of Theorem 5.1 is then concluded arguing by linearity. $\square$

REMARK 5.2. Let us comment on the difference between Assumption $\mathbf{P}$ and the set of hypotheses $A_{1}, \ldots, A_{N}$ appearing in the statement of Corollary 2.7. Assumption $\mathbf{P}$ is stronger in the sense that each $\hat{\gamma}_{j}$ is required to have empty intersection not only with $\gamma_{j-1}$ and $\gamma_{j+1}$, but also all with $\gamma_{k}$ for $k \neq j$. Assumption $\mathbf{P}$ guarantees that while inducing a transition between the levels $j$ and $j+1$ the other energy levels are untouched.

6. Genericity. We discuss in this section the genericity of Assumption $A_{j}$ which appears in Theorem 2.5 and Corollary 2.7. Recall that, by the Wigner-von Neumann theorem [44], the set of $N \times N$ Hermitian matrices of rank equal to $N-1$ (i.e., with one degenerate eigenvalue of multiplicity 2 and all other eigenvalues simple) is the finite union of submanifolds of codimension 3. More generally the set $\Upsilon$ of all $N \times N$ Hermitian matrices with degenerate eigenvalues is a Whitney stratified set (see for instance [25, Section 1.2]) of codimension 3. Similarly, symmetric real matrices with degenerate eigenvalues form a Whitney stratified set of codimension 2 of the space of all symmetric matrices. Let $d=3, H: \mathbf{U} \rightarrow \operatorname{Herm}(N)$ be a $\mathcal{C}^{3}$ map and $h=H(u) \in \operatorname{Herm}(N)$ have rank equal to $N-1$. Then $H(u)$ intersects $\Upsilon$ transversally at $h=H(u)$ if and only if $u$ is a conical intersection. By standard transversality arguments (see, for instance, [1, Proposition 19.1] and [25, Section 1.3.2]), it follows that there exists a residual set $\mathcal{R}$ in $\mathcal{C}^{3}(\mathbf{U}, \operatorname{Herm}(N))$ such that for every $H \in \mathcal{R}$, all intersections between the eigenvalues of $H$ are conical. (In particular for every $H \in \mathcal{R}$ and every $u \in \mathbf{U}$ such that $H(u) \in \Upsilon$, the rank of $H(u)$ is $N-1$, since the strata of $\Upsilon$ corresponding to matrices of lower rank are of codimension larger than 3.)

Moreover, a conical intersection is structurally stable in the sense that, if $u$ is a conical intersection for $H \in \mathcal{C}^{3}(\mathbf{U}, \operatorname{Herm}(N))$, then any small perturbation of $H$ has a conical intersection near $u$. Similar results hold for $d=2$ in the case of real symmetric Hamiltonians.

The following two results, whose proof can be directly derived from the above considerations, establish that a small one-parameter perturbation of a Hamiltonian with conically connected spectrum satisfies the assumptions of Theorem 2.5 and Corollary 2.7. Here we impose $\mathbf{U}$ to be bounded and the Hamiltonian $H$ to be $\mathcal{C}^{3}$ on $\overline{\mathbf{U}}$, meaning that it admits a $\mathcal{C}^{3}$ extension on a neighborhood of $\overline{\mathbf{U}}$. We also require $H$ not to have eigenvalue intersections on $\partial \mathbf{U}$. This prevents the occurrence of a sequence of eigenvalue intersections converging to $\partial \mathbf{U}$.

TheOREM 6.1. Let $\mathbf{U}$ be an open, connected and bounded subset of $\mathbb{R}^{3}$. Let $H: \overline{\mathbf{U}} \rightarrow \operatorname{Herm}(N)$ be a $\mathcal{C}^{3}$ map and $\left(\lambda_{j}(u)\right)_{j \in \llbracket 1, N \rrbracket}$ be the increasing sequence of eigenvalues of $H(u), u \in \overline{\mathbf{U}}$, repeated according to their multiplicities. Fix $j \in \llbracket 1, N-1 \rrbracket$. Assume that the levels $\lambda_{j}$ and $\lambda_{j+1}$ intersect and that all intersections between them are conical and correspond to controls $u \in \mathbf{U}$. If $j>1$ (respectively, $j<N-1$ ), assume, moreover, that all intersections between the levels $\lambda_{j-1}$ and $\lambda_{j}$ (respectively, $\lambda_{j+1}$ and $\lambda_{j+2}$ ) are conical and correspond to controls $u \in \mathbf{U}$.

Let us define

$$
\Xi=\left\{h \in \mathcal{C}^{3}(\overline{\mathbf{U}} \times \mathbb{R}, \operatorname{Herm}(N)) \mid h(\cdot, 0)=H(\cdot)\right\}
$$

endowed with the $\mathcal{C}^{3}$ Whitney topology induced by $\mathcal{C}^{3}(\overline{\mathbf{U}} \times \mathbb{R}, \operatorname{Herm}(N))$. 
Then there exists an open and dense subset $\hat{\Xi}$ of $\Xi$, such that for every $h \in \hat{\Xi}$, the restriction $h: \mathbf{U} \times[-\delta, \delta] \rightarrow \operatorname{Herm}(N)$ satisfies Assumption $A_{j}$ for some $\delta>0$.

Corollary 6.2. Let $\mathbf{U}$ be an open, connected and bounded subset of $\mathbb{R}^{3}$. Let $H: \overline{\mathbf{U}} \rightarrow \operatorname{Herm}(N)$ be a $\mathcal{C}^{3}$ map and $\left(\lambda_{j}(u)\right)_{j \in \llbracket 1, N \rrbracket}$ be the increasing sequence of eigenvalues of $H(u), u \in \overline{\mathbf{U}}$, repeated according to their multiplicities. Assume that all eigenvalue intersections are conical and correspond to controls $u \in \mathbf{U}$. Assume moreover that, for every $j \in \llbracket 1, N-1 \rrbracket, \lambda_{j}$ and $\lambda_{j+1}$ intersect.

Let $\Xi$ be defined as in Theorem 6.1. Then there exists a open and dense subset $\hat{\Xi}$ of $\Xi$, such that for every $h \in \hat{\Xi}$, the restriction $h: \mathbf{U} \times[-\delta, \delta] \rightarrow \operatorname{Herm}(N)$ satisfies Assumption $\mathbf{P}$ for some $\delta>0$ (and, in particular, Assumption $A_{j}$ every $j \in \llbracket 1, N \rrbracket$ ).

As a consequence of Corollary 2.7 and Theorem 5.1, every $h$ in the set $\hat{\Xi}$ of parameter-dependent Hamiltonians appearing in the statement of Corollary 6.2 defines, when restricted to $\mathbf{U} \times[-\delta, \delta]$, a system which is ensemble approximately controllable between eigenstates and which satisfies the conclusion of Theorem 5.1.

The same conclusions as those of Theorem 6.1 and Corollary 6.2 hold when $d=2$ (i.e., $\mathbf{U}$ is a subset of $\mathbb{R}^{2}$ ) and the Hamiltonians $H$ and $h$ take values in the set of symmetric $N \times N$ real matrices.

7. Multidimensional set of parameters. We consider in this section the situation in which the parameter on which the Hamiltonian depends varies in a set of dimension larger than one. Our technique still applies when the set of eigenvalue intersections projects onto a one-dimensional curve in the space of controls. Even if this situation is not generic, it however shows up in several physical examples, as we illustrate in Sections 7.1 and 7.2.

Let $\Sigma$ be the set of parameters, contained in $\mathbb{R}^{m}$ for some $m \in \mathbb{N}$. We consider the ensemble controllability problem for the Schrödinger equation on $\mathbb{C}^{N}, N \in \mathbb{N}$,

$$
i \dot{\psi}=H^{\sigma}(u(t)) \psi, \quad \sigma \in \Sigma .
$$

Denote by $\left(\lambda_{j}^{\sigma}(u)\right)_{j=1}^{N}$ the nondecreasing sequence of eigenvalues of $H^{\sigma}(u)$ repeated according to their multiplicities and by $\left(\phi_{1}^{\sigma}(u), \ldots, \phi_{N}^{\sigma}(u)\right)$ an orthonormal basis of associated eigenvectors. Let us define

$$
\begin{aligned}
\gamma_{0} & =\emptyset, \\
\gamma_{j} & =\left\{u \in \mathbf{U} \mid \exists \sigma \in \Sigma \text { such that } \lambda_{j}^{\sigma}(u)=\lambda_{j+1}^{\sigma}(u)\right\}, \quad j \in \llbracket 1, N-1 \rrbracket, \\
\gamma_{N} & =\emptyset .
\end{aligned}
$$

Assumption $A_{j}^{*}$. There exists a connected component $\hat{\gamma}_{j}$ of $\gamma_{j}$ such that

- There exists an interval $\left[\alpha_{0}, \alpha_{1}\right] \subset \mathbb{R}$ and a $\mathcal{C}^{3}$ embedding $\beta_{j}:\left[\alpha_{0}, \alpha_{1}\right] \rightarrow \mathbf{U}$ such that $\hat{\gamma}_{j}=\beta_{j}\left(\left[\alpha_{0}, \alpha_{1}\right]\right)$;

- $\hat{\gamma}_{j}$ is contained in $\mathbf{U} \backslash\left(\gamma_{j-1} \cup \gamma_{j+1}\right)$;

- For every $\sigma \in \Sigma$, there exists a unique $u \in \hat{\gamma}_{j}$ such that $\lambda_{j}^{\sigma}$ and $\lambda_{j+1}^{\sigma}$ have an intersection at $u$, which is conical.

Moreover the set $\mathbf{U} \backslash\left(\gamma_{j-1} \cup \gamma_{j} \cup \gamma_{j+1}\right)$ is pathwise connected.

Theorem 2.5 and Corollary 2.7 hold true by replacing $\left[\alpha_{0}, \alpha_{1}\right] \times \mathbf{U} \ni(\alpha, u) \mapsto$ $H^{\alpha}(u) \in \operatorname{Herm}(N)$ by $\Sigma \times \mathbf{U} \ni(\sigma, u) \mapsto H^{\sigma}(u) \in \operatorname{Herm}(N)$ and $A_{j}$ by $A_{j}^{*}$. The same proof works without major modifications.

7.1. Chirped pulses for two-level systems with two parameters. We consider here below an extension of the example studied in Section 4, in which we add 
an uncertain parameter also in the coupling between the control and the system. Consider the controlled equation

$$
i \frac{d}{d t}\left(\begin{array}{c}
\psi_{1}^{\sigma} \\
\psi_{2}^{\sigma}
\end{array}\right)=\left(\begin{array}{cc}
E+\alpha & \beta \Omega(t) \\
\beta \Omega^{*}(t) & -E-\alpha
\end{array}\right)\left(\begin{array}{c}
\psi_{1}^{\sigma} \\
\psi_{2}^{\sigma}
\end{array}\right),
$$

where $\sigma=(\alpha, \beta) \in\left[\alpha_{0}, \alpha_{1}\right] \times\left[\beta_{0}, \beta_{1}\right] \subset \mathbb{R} \times(0,+\infty)$. As before, $E>0$ is fixed and we want to steer the system from $\phi_{1}^{\sigma}(0)=(1,0)$ to $\phi_{2}^{\sigma}(0)=(0,1)$, up to phases.

The same change of variables as in Section 4 and the control trasformation

$$
\Omega(t)=u_{1}(t) e^{-i(2 E t+\Delta(t))}, \quad u_{2}(t)=\frac{d \Delta}{d t}(t),
$$

yield the equivalent system $i \frac{d}{d t} \Phi^{\sigma}=\mathcal{H}^{\sigma}(t) \Phi^{\sigma}$ with

$$
\mathcal{H}^{\sigma}(t)=\left(\begin{array}{cc}
\alpha & \beta u_{1}(t) \\
\beta u_{1}(t) & -\alpha+u_{2}(t)
\end{array}\right) .
$$

Since the eigenvalue intersections of $\mathcal{H}^{\sigma}$ only depend on $\alpha$ and not on $\beta$, the entire discussion of Section 4 still applies in the presence of the parameter $\beta$. This underlines once more the robustness of the chirped pulse strategy for two-level systems.

7.2. Example 2: STIRAP. Consider the three-level system with controlled Hamiltonian

$$
H^{\left(\alpha_{1}, \alpha_{3}, \beta_{1}, \beta_{2}\right)}\left(u_{1}, u_{2}\right)=\left(\begin{array}{ccc}
E_{1}+\alpha_{1} & \beta_{1} u_{1} & 0 \\
\beta_{1} u_{1} & E_{2} & \beta_{2} u_{2} \\
0 & \beta_{2} u_{2} & E_{3}+\alpha_{3}
\end{array}\right)
$$

with $\alpha_{j} \in\left[\alpha_{j 0}, \alpha_{j 1}\right], j=1,3, \beta_{j} \in\left[\beta_{j 0}, \beta_{j 1}\right], j=1,2, \beta_{10}, \beta_{20}>0$ and $\alpha_{j 0}, \alpha_{j 1}$ such that $E_{1}+\alpha_{1}<E_{2}<E_{3}+\alpha_{3}$. (One could clearly add a further parameter uncertainty in the level $E_{2}$, which is not relevant, since the trace of the matrix can always be shifted without modifying the dynamical properties of the system.) The controls $u_{1}$ and $u_{2}$ are real-valued.

Denote $\sigma=\left(\alpha_{1}, \alpha_{3}, \beta_{1}, \beta_{2}\right)$ and

$$
\Sigma=\left[\alpha_{10}, \alpha_{11}\right] \times\left[\alpha_{30}, \alpha_{31}\right] \times\left[\beta_{10}, \beta_{11}\right] \times\left[\beta_{20}, \beta_{21}\right] .
$$

The eigenvalue intersections of $H^{\sigma}\left(u_{1}, u_{2}\right)$ occur on the axes $u_{1}$ and $u_{2}$. More precisely,

$$
\begin{aligned}
\gamma_{1}= & \left\{\left(0,-\frac{\sqrt{\left(E_{1}+\alpha_{1}-E_{3}-\alpha_{3}\right)\left(E_{1}+\alpha_{1}-E_{2}\right)}}{\beta_{2}}\right) \mid \sigma \in \Sigma\right\} \cup \\
& \left\{\left(0, \frac{\sqrt{\left(E_{1}+\alpha_{1}-E_{3}-\alpha_{3}\right)\left(E_{1}+\alpha_{1}-E_{2}\right)}}{\beta_{2}}\right) \mid \sigma \in \Sigma\right\}, \\
\gamma_{2}= & \left\{\left(-\frac{\sqrt{\left(E_{1}+\alpha_{1}-E_{3}-\alpha_{3}\right)\left(E_{2}-E_{3}-\alpha_{3}\right)}}{\beta_{1}}, 0\right) \mid \sigma \in \Sigma\right\} \cup \\
& \left\{\left(\frac{\sqrt{\left(E_{1}+\alpha_{1}-E_{3}-\alpha_{3}\right)\left(E_{2}-E_{3}-\alpha_{3}\right)}}{\beta_{1}}, 0\right) \mid \sigma \in \Sigma\right\} .
\end{aligned}
$$

In particular, $\gamma_{1}$ and $\gamma_{2}$ are both unions of two segments (see Figure 7.1), hence they are 1-dimensional smooth embedded manifolds. All hypotheses of Corollary 2.7 


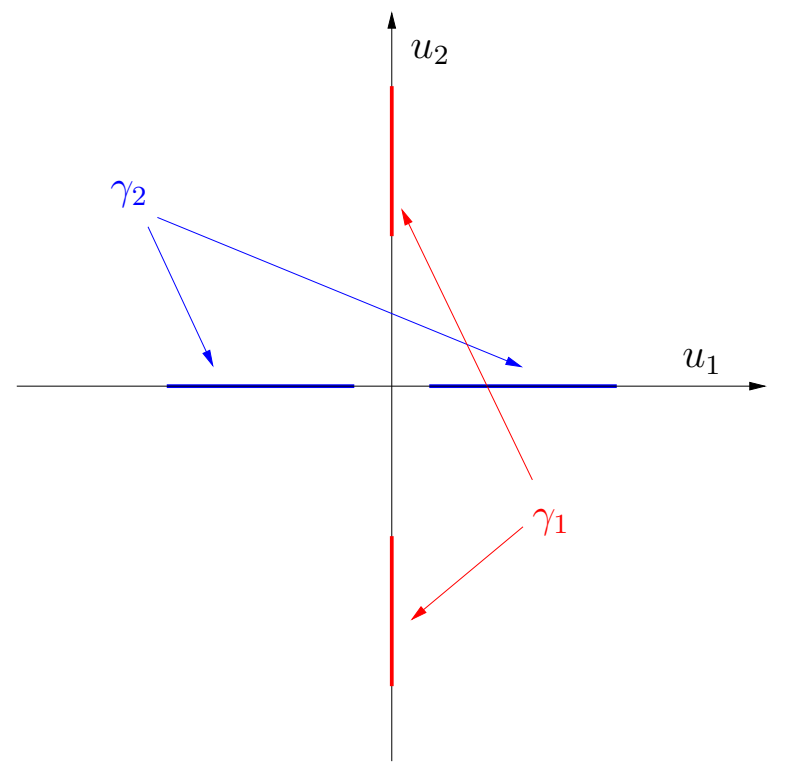

FIG. 7.1. The sets $\gamma_{1}$ and $\gamma_{2}$ for the STIRAP process.

(in the multi-dimensional parameter extension discussed above) are satisfied and we conclude that the system is ensemble approximately controllable between eigenstates. Moreover, according to Remark 2.8, the precision of the transition described in Theorem 2.5 can be made of order $\varepsilon$, where $\varepsilon$ is the velocity at which we follow the adiabatic path.

For instance, in order to steer system $(7.1)$ from $\phi_{1}^{\sigma}=(1,0,0)$ to $\phi_{3}^{\sigma}=(0,0,1)$ we can follow the path in Figure 7.2a.

In Figure $7.2 \mathrm{~b}$ we plot the two components of the control realising the transition. Notice that such controls are in the celebrated counter-intuitive order, meaning that, in order to go from state 1 to state 3 , one first activates the control $u_{2}$, responsible for the transition $2 \rightarrow 3$, and then the control $u_{1}$, responsible for the transition $1 \rightarrow 2$ (creating the so-called dark state). The approach presented in this paper gives a complete mathematical explanation of why the counter-intuitive order works and why it is so robust with respect to parameter fluctuations.

8. Extension to the infinite-dimensional case. The results of the previous sections extend, under some suitable regularity assumptions, to the case where $\mathbb{C}^{N}$ is replaced by an infinite-dimensional complex separable Hilbert space $\mathcal{H}$.

In order to avoid excessive technicalities, we present this extension in the case where the Hamiltonian $H$ depends affinely on the controls and where the controlled Hamiltonians are bounded. (For the general nonlinear case, one could follow the approach in [16].)

We then consider a Hamiltonian of the type

$$
H^{\sigma}(u)=H_{0}^{\sigma}+\sum_{j=1}^{d} u_{j} H_{j}^{\sigma}
$$

wih the parameter $\sigma$ belonging to $\Sigma \subset \mathbb{R}^{m}$ for some $m \in \mathbb{N}$, and with $H_{j}^{\sigma}, j=0, \ldots, d$, satisfying the following assumption. 


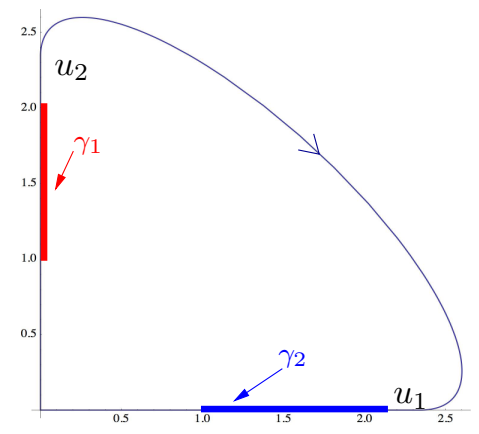

(a)

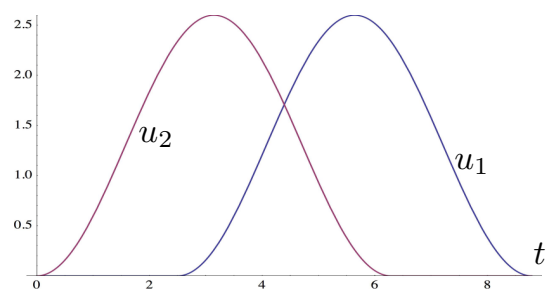

(b)

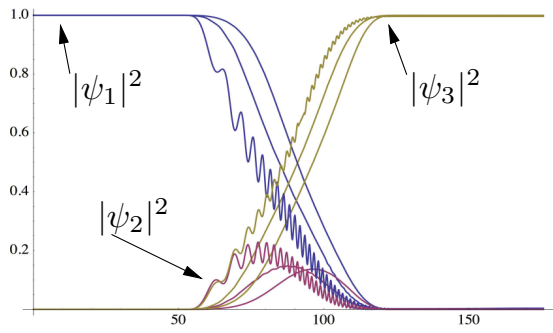

(c)

FIG. 7.2. Example of STIRAP process with $E_{1}=-1, E_{2}=0, E_{3}=1, \alpha_{1}, \alpha_{3} \in[-0.1,0.1]$, $\beta_{1}, \beta_{2} \in[0.8,1.2]$. In (a) we give the parametric plot of the control, in (b) the shape of its components as functions of time and in (c) the components of the wave function for the three choices of parameters $\left(\alpha_{1}, \alpha_{3}, \beta_{1}, \beta_{2}\right)=(0,0,1,1),(0.1,-0.1,0.8,1.2),(-0.1,0.1,1.2,0.8)$. For this simulation we used $\varepsilon=0.05$.

$\left(\mathbf{H}_{\infty}\right)$ Fix $\sigma_{0} \in \Sigma$ and assume that:

- $H_{0}^{\sigma_{0}}$ is self-adjoint and bounded from below;

- $H_{0}^{\sigma}-H_{0}^{\sigma_{0}}, H_{1}^{\sigma}, \ldots, H_{d}^{\sigma}$ are bounded for all $\sigma \in \Sigma$;

- the map $(\sigma, u) \mapsto H_{0}^{\sigma_{0}}-H^{\sigma}(u)$ is $\mathcal{C}^{3}$ from $\Sigma \times \mathbf{U}$ to the Banach space of bounded self-adjoint operators endowed with the operator norm;

- for all $(\sigma, u) \in \Sigma \times \mathbf{U}$ the eigenvalues $\lambda_{1}^{\sigma}(u) \leq \cdots \leq \lambda_{N}^{\sigma}(u)$ of $H^{\sigma}(u)$ are such that

○ $\operatorname{spectrum}\left(H^{\sigma}(u)\right) \cap\left[\lambda_{1}^{\sigma}(u), \lambda_{N}^{\sigma}(u)\right]=\left\{\lambda_{1}^{\sigma}(u), \ldots, \lambda_{N}^{\sigma}(u)\right\}$ and $\lambda_{j}^{\sigma}(u)$ has finite multiplicity for every $j \in \llbracket 1, N \rrbracket$,

○ $\operatorname{dist}\left(\operatorname{spectrum}\left(H^{\sigma}(u)\right) \backslash\left[\lambda_{1}^{\sigma}(u), \lambda_{N}^{\sigma}(u)\right],\left\{\lambda_{1}^{\sigma}(u), \lambda_{N}^{\sigma}(u)\right\}\right) \geq \Gamma$, with $\Gamma>0$ independent of $(\sigma, u)$ (see Figure 8.1).

Under the hypothesis $\left(\mathbf{H}_{\infty}\right)$, for every continuous control $u:[0, T] \rightarrow \mathbf{U}$ and every initial condition, the equation

$$
i \dot{\psi}=H^{\sigma}(u(t)) \psi
$$

admits a unique solution [43, Proposition 2.1].

Let $P^{\sigma, u}: \mathcal{H} \rightarrow \mathcal{H}$ be the orthogonal projector onto the sum of the eigenspaces corresponding to $\lambda_{1}^{\sigma}(u), \ldots, \lambda_{N}^{\sigma}(u)$. Without loss of generality this space is of complex dimension $N$. Then (see, for instance, [37]), for every $(\bar{\sigma}, \bar{u}) \in \Sigma \times \mathbf{U}$ and every $(\sigma, u)$ 


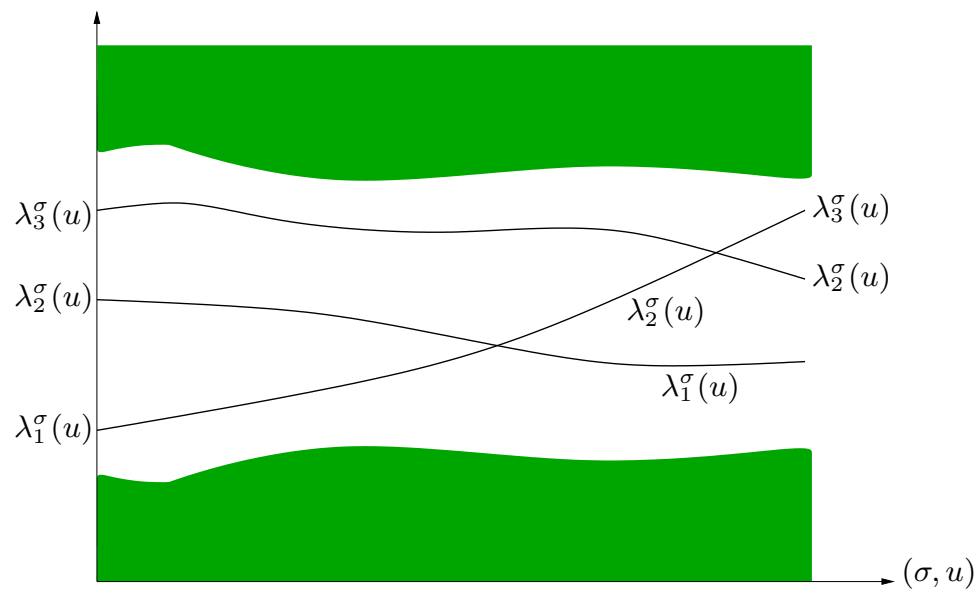

FIG. 8.1. The eigenvalues $\lambda_{1}^{\sigma}(u) \leq \lambda_{2}^{\sigma}(u) \leq \lambda_{3}^{\sigma}(u)$ of $H^{\sigma}(u)$ separated from the rest of the spectrum (which is contained in the shaded regions).

in a neighborhood of $(\bar{\sigma}, \bar{u})$,

$$
P^{\sigma, u}(\psi)=-\frac{1}{2 \pi i} \int_{\gamma}\left(H^{\sigma}(u)-c\right)^{-1} d c,
$$

where $\gamma$ is a Jordan curve in $\mathbb{C}$ separating $\lambda_{1}^{\bar{\sigma}}(\bar{u}), \ldots, \lambda_{N}^{\bar{\sigma}}(\bar{u})$ and $\operatorname{spectrum}\left(H^{\bar{\sigma}}(\bar{u})\right) \backslash$ $\left[\lambda_{1}^{\bar{\sigma}}(\bar{u}), \lambda_{N}^{\bar{\sigma}}(\bar{u})\right]$. Hence $(\sigma, u) \mapsto P^{\sigma, u}$ is $\mathcal{C}^{3}$ as a map from $\Sigma \times \mathbf{U}$ to the Banach space of bounded operators on $\mathcal{H}$ endowed with the operator norm.

Let $I^{\sigma, u}: \mathbb{C}^{N} \rightarrow \mathcal{H}$ be a linear map such that $I^{\sigma, u}$ is unitary between $\mathbb{C}^{N}$ and the image of $P^{\sigma, u}$.

Assume for now that $(\sigma, u) \mapsto I^{\sigma, u}$ is globally $\mathcal{C}^{3}$ on $\Sigma \times \mathbf{U}$. We denote by $\left(I^{\sigma, u}\right)^{-1}$ the inverse of $I^{\sigma, u}$ on the image of $P^{\sigma, u}$. The Hamiltonian $(\sigma, u) \mapsto \widehat{H}^{\sigma}(u)=$ $\left(I^{\sigma, u}\right)^{-1} H^{\sigma}(u) I^{\sigma, u} \in \operatorname{Herm}(N)$ is $\mathcal{C}^{3}$ and its eigenvalues are $\lambda_{1}^{\sigma}(u), \ldots, \lambda_{N}^{\sigma}(u)$.

A general adiabatic theorem (see, for instance, [43, Theorem 2.2]) states that, given a $\mathcal{C}^{2}$ path $u:[0,1] \rightarrow \mathbf{U}$ and $\hat{\psi}_{0} \in \mathbb{C}^{N}$, the solutions $\psi(\cdot)$ and $\hat{\psi}(\cdot)$ of, respectively, $i \dot{\psi}(t)=H^{\sigma}(u(\varepsilon t)) \psi(t), \psi(0)=\left(I^{\sigma, u(0)}\right)^{-1} \hat{\psi}_{0}$, and $i \dot{\hat{\psi}}(t)=\widehat{H}^{\sigma}(u(\varepsilon t)) \hat{\psi}(t), \hat{\psi}(0)=\hat{\psi}_{0}$, are such that $\psi(1 / \varepsilon)$ is $\varepsilon$-close, up to phases, to $\left(I^{\sigma, u(1)}\right)^{-1} \hat{\psi}(1 / \varepsilon)$.

When $(\sigma, u) \mapsto I^{\sigma, u}$ cannot be globally defined as a $\mathcal{C}^{3}$ map, the same arguments can be applied in local charts leading to the following generalization of Theorem 2.5 and Corollary 2.7

As in the previous section, the sets $\gamma_{j}$ are defined as

$$
\begin{aligned}
\gamma_{0} & =\emptyset, \\
\gamma_{j} & =\left\{u \in \mathbf{U} \mid \exists \sigma \in \Sigma \text { such that } \lambda_{j}^{\sigma}(u)=\lambda_{j+1}^{\sigma}(u)\right\}, \quad j \in \llbracket 1, N-1 \rrbracket, \\
\gamma_{N} & =\emptyset .
\end{aligned}
$$

Theorem 8.1. Assume that $\left(H_{\infty}\right)$ holds true. Let $j \in \llbracket 1, N-1 \rrbracket$ be such that Assumption $A_{j}^{*}$ of Section 7 is satisfied. Take $u_{0}, u_{1} \in \mathbf{U} \backslash\left(\gamma_{j-1} \cup \gamma_{j} \cup \gamma_{j+1}\right)$ and consider a $\mathcal{C}^{3}$ path $u(\cdot):[0,1] \rightarrow \mathbf{U}$ satisfying $u(0)=u_{0}, u(1)=u_{1}$, and such that $\left.u\right|_{\left[t_{0}, t_{1}\right]}$ is a reparameterization of $\beta_{j}$ for some $0<t_{0}<t_{1}<1$ such that $u(t) \notin$ $\gamma_{j-1} \cup \gamma_{j} \cup \gamma_{j+1}$ for every $t \in[0,1] \backslash\left[t_{0}, t_{1}\right]$. Assume, moreover, that $\dot{u}(t) \neq 0$ for every $t \in[0,1]$. 
Then there exists a constant $C>0$ such that for every $\sigma \in \Sigma$ and $\varepsilon>0$ the solutions $\psi_{\varepsilon}^{\sigma, \pm}$ of

$$
i \dot{\psi}_{\varepsilon}^{\sigma, \pm}(t)=H^{\sigma}(u(\varepsilon t)) \psi_{\varepsilon}^{\sigma, \pm}(t)
$$

with initial conditions

$$
\psi_{\varepsilon}^{\sigma,+}(0)=\phi_{j}^{\sigma}\left(u_{0}\right), \quad \psi_{\varepsilon}^{\sigma,-}(0)=\phi_{j+1}^{\sigma}\left(u_{0}\right)
$$

satisfy

$$
\left\|\psi_{\varepsilon}^{\sigma,+}(1 / \varepsilon)-e^{i \theta^{+}} \phi_{j+1}^{\sigma}\left(u_{1}\right)\right\| \leq C \sqrt{\varepsilon}, \quad\left\|\psi_{\varepsilon}^{\sigma,-}(1 / \varepsilon)-e^{i \theta^{-}} \phi_{j}^{\sigma}\left(u_{1}\right)\right\| \leq C \sqrt{\varepsilon},
$$

for some $\theta^{ \pm} \in \mathbb{R}$.

Corollary 8.2. Assume that $\left(H_{\infty}\right)$ holds true. Let Assumption $A_{j}^{*}$ be satisfied for every $j \in \llbracket 1, N-1 \rrbracket$. Then (8.1) is ensemble approximately controllable between the eigenstates $\left\{\phi_{1}^{\sigma}(u), \ldots, \phi_{N}^{\sigma}(u)\right\}$ in the sense that for every $\varepsilon>0, j, k \in \llbracket 1, N \rrbracket$ and $u_{0}, u_{1} \in \mathbf{U}$ such that $\lambda_{j}^{\sigma}\left(u_{0}\right)$ and $\lambda_{k}^{\sigma}\left(u_{1}\right)$ are simple for every $\sigma \in \Sigma$, there exists a control $u(\cdot):[0, T] \rightarrow \mathbf{U}$ such that for every $\sigma \in \Sigma$ the solution of (8.1) with initial condition $\psi^{\sigma}(0)=\phi_{j}^{\sigma}\left(u_{0}\right)$ satisfies $\left\|\psi^{\sigma}(T)-e^{i \theta} \phi_{k}^{\sigma}\left(u_{1}\right)\right\|<\varepsilon$ for some $\theta \in \mathbb{R}$.

REMARK 8.3. When $\Sigma$ is one-dimensional, genericity results similar to those in Section 6 can be obtained. For related structural stability and genericity considerations, see [9].

8.1. Example 3: Eberly-Law-like models. We study in this section a model for coupled spin-oscillator dynamics (see [30, 45, 7]). For other spin-boson models, see $[11,29,36]$ and references therein. The state space is the tensor product of the state spaces of an harmonic oscillator and of a spin- $\frac{1}{2}$ particle. The system has two control parameters that we assume to be real, the first one, $u_{1}$, coupling the two levels of the spin system and the other one, $u_{2}$, producing simultaneous spin transitions and vibrational phonon excitations.

We let $\omega$ be the gap between the levels of the harmonic oscillator and $\delta$ be the internal gap of the spin system, $\alpha$ (respectively, $\beta$ ) the coupling strength between $u_{1}$ (respectively, $\left.u_{2}\right)$ and the system. The vector $\sigma=(\alpha, \beta, \omega, \delta)$ will be considered as a set of parameters whose uncertainty will be tackled by the technique proposed in the previous sections.

The model can then be represented by the infinite-dimensional controlled quantum system

$$
i \dot{\psi}=H^{\sigma}(u) \psi, \quad \psi \in \ell^{2},
$$

where $u=\left(u_{1}, u_{2}\right) \in \mathbb{R}^{2}, \sigma \in\left[\alpha_{0}, \alpha_{1}\right] \times\left[\beta_{0}, \beta_{1}\right] \times\left[\omega_{0}, \omega_{1}\right] \times\left[\delta_{0}, \delta_{1}\right]=\Sigma$, and

$$
H^{\sigma}(u)=\left(\begin{array}{cccccc}
0 & \alpha u_{1} & 0 & 0 & 0 & \cdots \\
\alpha u_{1} & \delta & \beta u_{2} & 0 & 0 & \ddots \\
0 & \beta u_{2} & \omega & \alpha u_{1} & 0 & \ddots \\
0 & 0 & \alpha u_{1} & \omega+\delta & \beta u_{2} & \ddots \\
0 & 0 & 0 & \beta u_{2} & 2 \omega & \ddots \\
\vdots & \ddots & \ddots & \ddots & \ddots & \ddots
\end{array}\right) .
$$


We assume that $\alpha_{0}, \beta_{0}, \omega_{0}, \delta_{0}>0$. For simplicity, we also assume that $\omega_{0}>\delta_{1}$, that is, the diagonal of $H^{\sigma}(u)$ is a strictly increasing sequence. Further conditions on $\alpha_{j}, \beta_{j}, \omega_{j}, \delta_{j}, j=0,1$, will be imposed below (see (8.3)).

The Hamiltonian $H^{\sigma}(u)$ is self-adjoint, has purely discrete spectrum, and, if both $u_{1}$ and $u_{2}$ are different from zero, then all eigenvalues of $H^{\sigma}(u)$ are non-degenerate (see [2]).

In order to apply our general strategy, let us describe the eigenvalue intersections of $H^{\sigma}(u)$. This is quite simple, since, for $u_{1}=0$ or $u_{2}=0$, the matrix describing $H^{\sigma}(u)$ is block-diagonal.

Let us first consider intersections along the axis $u_{2}=0$. The eigenvalues of $H^{\sigma}\left(u_{1}, 0\right)$ as a function of $u_{1}$ are shown in Figure 8.2. A simple computation shows that the smallest value of $\left|u_{1}\right|$ for which $H^{\sigma}\left(u_{1}, 0\right)$ has degenerate eigenvalues is $u_{1}^{*}=$ $\frac{\sqrt{\omega^{2}-\delta^{2}}}{2 \alpha}$ and the second-smallest value is $\bar{u}_{1}=\frac{\sqrt{4 \omega^{2}-\delta^{2}}}{2 \alpha}$.

We reason similarly along the axis $u_{1}=0$ and we get that the smallest value of $\left|u_{2}\right|$ for which $H^{\sigma}\left(0, u_{2}\right)$ has degenerate eigenvalues different from zero is $u_{2}^{*}=\frac{\sqrt{\delta(2 \omega-\delta)}}{2 \beta}$ and the second-smallest value is $\bar{u}_{2}=\frac{\sqrt{\delta(2 \omega-\delta)+3 \omega^{2}}}{2 \beta}$. The smallest value of $\left|u_{2}\right|$ for which 0 is a degenerate eigenvalue for $H^{\sigma}\left(0, u_{2}\right)$ is $u_{20}^{*}=\frac{\sqrt{\delta \omega}}{\beta}$ and the second-smallest value is $\bar{u}_{20}=\frac{\sqrt{2 \omega(\delta+\omega)}}{\beta}$.
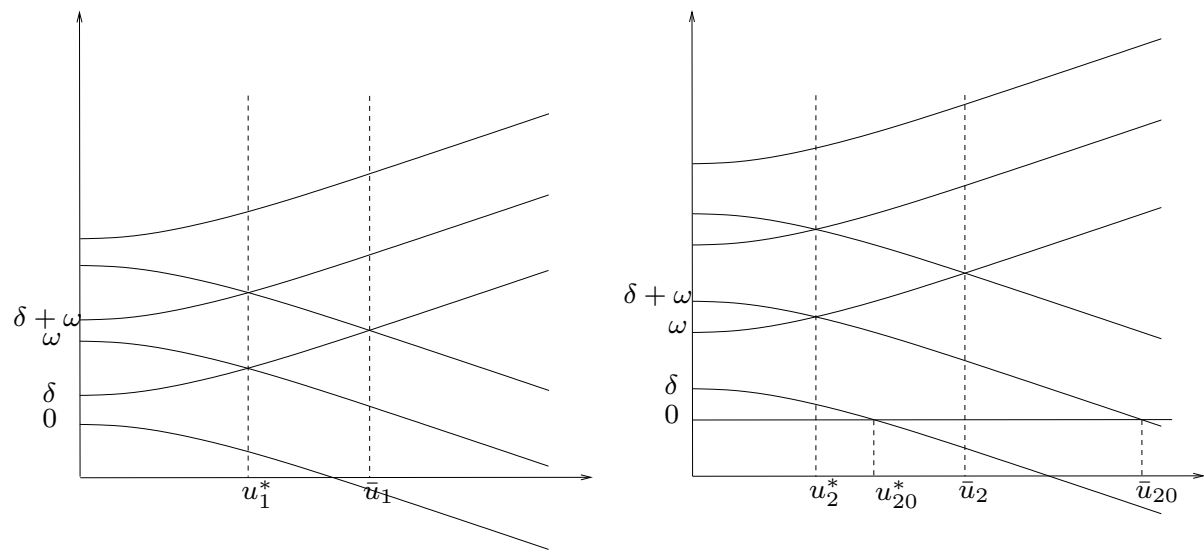

FIG. 8.2. The eigenvalues of $H^{\sigma}\left(u_{1}, 0\right)$ (left) and $H^{\sigma}\left(0, u_{2}\right)$ (right).

We assume in the following that

$$
\max _{\sigma \in \Sigma} u_{1}^{*}<\min _{\sigma \in \Sigma} \bar{u}_{1}, \quad \max _{\sigma \in \Sigma} u_{2}^{*}<\min _{\sigma \in \Sigma} \bar{u}_{2}, \quad \max _{\sigma \in \Sigma} u_{20}^{*}<\min _{\sigma \in \Sigma} \bar{u}_{20} .
$$

Let $U=\left[-\eta, \eta+\max _{\sigma \in \Sigma} u_{1}^{*}\right] \times\left[-\eta, \eta+\max _{\sigma \in \Sigma} u_{20}^{*}\right]$ with $\eta>0$ small. Then Assumptions $A_{j}^{*}, j \geq 1$, introduced in Section 7, hold true with

$$
\begin{aligned}
\hat{\gamma}_{1} & =\{0\} \times\left[\min _{\sigma \in \Sigma} u_{20}^{*}, \max _{\sigma \in \Sigma} u_{20}^{*}\right], & & \\
\hat{\gamma}_{2 j+1} & =\{0\} \times\left[\min _{\sigma \in \Sigma} u_{2}^{*}, \max _{\sigma \in \Sigma} u_{2}^{*}\right], & & j \geq 1, \\
\hat{\gamma}_{2 j} & =\left[\min _{\sigma \in \Sigma} u_{1}^{*}, \max _{\sigma \in \Sigma} u_{1}^{*}\right] \times\{0\}, & & j \geq 1 .
\end{aligned}
$$

In particular, all eigenvalue intersections in $\hat{\gamma}_{j}, j \geq 1$, are conical. Condition (8.3) also implies that, for every $n \in \mathbb{N}$, Assumption $\left(\mathbf{H}_{\infty}\right)$ is satisfied by the first $2 n+1$ 
eigenvalues with $\mathbf{U}=U \cap\left\{u_{2}<u_{1}+\eta\right\}$, by the first 2 eigenvalues $\lambda_{1}^{\sigma}, \lambda_{2}^{\sigma}$ with $\mathbf{U}=U \cap\left\{u_{1}<u_{2}+\eta\right\}$ and by the eigenvalues $\lambda_{3}^{\sigma}, \ldots, \lambda_{2 n}^{\sigma}$ with $\mathbf{U}=U \cap\left\{u_{1}<\right.$ $\left.u_{2}+\eta, u_{2}<\max _{\sigma \in \Sigma} u_{2}^{*}+\eta\right\}$.

Henceforth, ensemble approximate controllability between eigenvectors holds true. Figure 8.3 shows a path in the space of controls leading to an ensemble transfer from $\phi_{1}^{\sigma}(0)$ to $\phi_{4}^{\sigma}(0)$. The path is a loop starting and ending at $u=0$ that goes through $\hat{\gamma}_{1}, \hat{\gamma}_{2}$, and $\hat{\gamma}_{3}$.

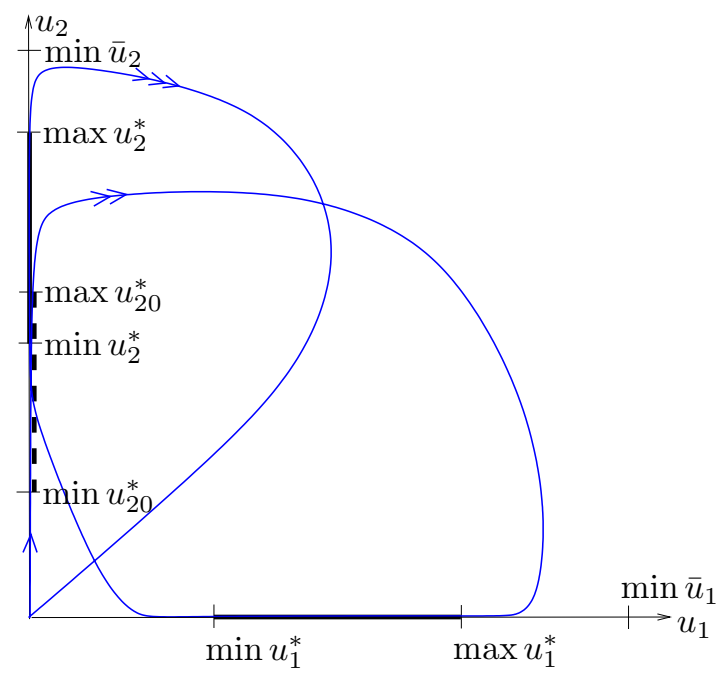

FIG. 8.3. A loop in the plane $\left(u_{1}, u_{2}\right)$ inducing a population transfer from the first to the fourth eigenstate of the drift Hamiltonian.

Appendix A. Regularity of eigenvalues and eigenvectors. In this section we prove Lemma 3.2, which states that a time-dependent Hamiltonian $H \in$ $\mathcal{C}^{k+1}(I, \operatorname{Herm}(N))$ whose eigenvalues intersections are all conical has $\mathcal{C}^{k+1}$ eigenvalues and $\mathcal{C}^{k}$ eigenvectors.

Proof of Lemma 3.2. For each $j \in \llbracket 1, N \rrbracket$, consider the function

$$
\ell_{j}: \operatorname{Herm}(N) \rightarrow \mathbb{R}
$$

that associates with a Hermitian matrix its $j$-th eigenvalue, where eigenvalues are counted according to their multiplicities, in such a way that $\ell_{1} \leq \cdots \leq \ell_{N}$. By Rellich theorem [38], each map $\ell_{j}$ is analytic on

$$
\operatorname{Herm}_{j}(N)=\left\{h \in \operatorname{Herm}(N) \mid \ell_{j}(h) \text { is a simple eigenvalue of } h\right\} .
$$

Moreover, for every $h \in \operatorname{Herm}_{j}(N)$, there exist a neighborhood $\mathcal{V}_{h}$ of $h$ in $\operatorname{Herm}_{j}(N)$ and an analytic function $\varphi_{j}: \mathcal{V}_{h} \rightarrow \mathbb{C}^{N}$ such that $\varphi_{j}(g)$ is a norm 1 eigenvector of $g$ of eigenvalue $\ell_{j}(g)$ for every $g \in \mathcal{V}_{h}$. Hence, for every $j \in \llbracket 1, N \rrbracket$, the $j$-th eigenvalue and a choice of a corresponding eigenvector of norm 1 are $\mathcal{C}^{k+1}$ on

$$
\left\{t \in I \mid \ell_{j}(H(t)) \text { is a simple eigenvalue of } H(t)\right\} .
$$

We are left to asses the regularity of eigenpairs in a neighborhood of a time $\bar{t} \in I$ such that $H(\bar{t}) \notin \operatorname{Herm}_{j}(N)$. 
Let $J$ be a neighborhood of $\bar{t}$ in $I$ such that for every $t \in J \backslash\{\bar{t}\}$ both $\ell_{j}(H(t))$ and $\ell_{j+1}(H(t))$ are simple. For every $t \in J$, let $P^{t}: \mathbb{C}^{N} \rightarrow \mathbb{C}^{N}$ be the orthogonal projector on the sum of the eigenspaces corresponding to $\ell_{j}(H(t))$ and $\ell_{j+1}(H(t))$.

Then (see, for instance, [37]),

$$
P^{t}=-\frac{1}{2 \pi i} \int_{\gamma}(H(t)-c)^{-1} d c
$$

where $\gamma$ is a Jordan curve in $\mathbb{C}$ separating $\ell_{j}(H(t)), \ell_{j+1}(H(t))$ and

$$
\operatorname{spectrum}(H(t)) \backslash\left\{\ell_{j}(H(t)), \ell_{j+1}(H(t))\right\} .
$$

Up to taking a smaller $J$ if necessary, the curve $\gamma$ can be taken independently of $t \in J$.

Hence $t \mapsto P^{t}$ is $\mathcal{C}^{k+1}$ as a map from $J$ to the space of linear operators on $\mathbb{C}^{N}$. Then there exists a linear map $I^{t}: \mathbb{C}^{2} \rightarrow \mathbb{C}^{N}$, depending $\mathcal{C}^{k+1}$ on $t$, such that $I^{t}$ is unitary between $\mathbb{C}^{2}$ and the image of $P^{t}$. We denote by $\left(I^{t}\right)^{-1}$ the inverse of $I^{t}$ on the image of $P^{t}$.

The Hamiltonian $h(t)=\left(I^{t}\right)^{-1} H(t) I^{t}: \mathbb{C}^{2} \rightarrow \mathbb{C}^{2}$ is well defined on $J$, depends $\mathcal{C}^{k+1}$ on $t$, and has $\ell_{j}(H(t))$ and $\ell_{j+1}(H(t))$ as eigenvalues. If, moreover, $v$ is an eigenvector of $h(t)$, then $I^{t} v$ is an eigenvector of $H(t)$. We are therefore left to prove the result for the eigenpairs of $h(t)$.

Without loss of generality we can consider the case where the trace of $h(t)$ is zero for every $t \in J$. Hence, $h(t)$ has the form

$$
h(t)=\left(\begin{array}{cc}
a(t) & b(t)+i c(t) \\
b(t)-i c(t) & -a(t)
\end{array}\right),
$$

where $a(\cdot), b(\cdot), c(\cdot)$ are real-valued $\mathcal{C}^{k+1}$ functions on $J$.

Moreover, since $h(\bar{t})$ has a double eigenvalue, we have $a(\bar{t})=b(\bar{t})=c(\bar{t})=0$. Without loss of generality $\bar{t}=0$.

Let $\alpha, \beta, \gamma: J \rightarrow \mathbb{R}$ be $\mathcal{C}^{k}$ functions such that

$$
a(t)=t \alpha(t), \quad b(t)=t \beta(t), \quad c(t)=t \gamma(t), \quad t \in J .
$$

Hypothesis (3.2) guarantees that $\alpha(0) \neq 0$ or $\beta(0) \neq 0$ or $\gamma(0) \neq 0$. Notice that, up to applying a unitary change of variables in $\mathbb{C}^{2}$ and restricting $J$, we can assume that $\beta(t) \neq 0$ for every $t \in J$.

The eigenvalues of $h(t)$ are $\pm \sqrt{a(t)^{2}+b(t)^{2}+c(t)^{2}}= \pm|t| \sqrt{\alpha(t)^{2}+\beta(t)^{2}+\gamma(t)^{2}}$. The function

$$
t \mapsto \begin{cases}-\sqrt{a(t)^{2}+b(t)^{2}+c(t)^{2}} & \text { if } t<0 \\ \sqrt{a(t)^{2}+b(t)^{2}+c(t)^{2}} & \text { if } t \geq 0\end{cases}
$$

is then equal to $t \sqrt{\alpha(t)^{2}+\beta(t)^{2}+\gamma(t)^{2}}$ which is $\mathcal{C}^{k+1}$ on $J$. Indeed, the only term of the $(k+1)$-th derivative of $t \sqrt{\alpha(t)^{2}+\beta(t)^{2}+\gamma(t)^{2}}$ involving the $(k+1)$-th derivative of $\alpha, \beta$, and $\gamma$ is

$$
\frac{t\left(\alpha^{(k+1)}(t) \alpha(t)+\beta^{(k+1)}(t) \beta(t)+\gamma^{(k+1)}(t) \gamma(t)\right)}{\sqrt{\alpha(t)^{2}+\beta(t)^{2}+\gamma(t)^{2}}} .
$$

Now, since $a(t)=t \alpha(t)$ is $\mathcal{C}^{k+1}$, the term $t \alpha^{(k+1)}(t)$ is continuous. The same argument holds for $\beta$ and $\gamma$ and the $\mathcal{C}^{k+1}$ regularity of $t \sqrt{\alpha(t)^{2}+\beta(t)^{2}+\gamma(t)^{2}}$ is proved. 
For what concerns the unit eigenvectors, a simple calculation shows that, up to phases and scaling, they are equal to

$$
\left(\frac{-a(t) \pm \sqrt{a(t)^{2}+b(t)^{2}+c(t)^{2}}}{b(t)-i c(t)}, 1\right)=\left(\frac{-\alpha(t) \pm \operatorname{sign}(t) \sqrt{\alpha(t)^{2}+\beta(t)^{2}+\gamma(t)^{2}}}{\beta(t)-i \gamma(t)}, 1\right)
$$

for $t \neq 0$. As above, connecting suitably the two branches, we can rewrite the eigenvectors as

$$
\left(\frac{-\alpha(t) \pm \sqrt{\alpha(t)^{2}+\beta(t)^{2}+\gamma(t)^{2}}}{\beta(t)-i \gamma(t)}, 1\right)
$$

which are $\mathcal{C}^{k}$ on $J$.

Acknowledgements: This work was supported by the project DISQUO of the DEFI InFIniTI 2017 by CNRS, by the ANR project SRGI ANR-15- CE40-0018, by the ANR project Quaco ANR-17-CE40-0007-01.

\section{REFERENCES}

[1] R. Abraham and J. Robbin. Transversal mappings and flows. An appendix by Al Kelley. W. A. Benjamin, Inc., New York-Amsterdam, 1967.

[2] R. Adami and U. Boscain. Controllability of the Schrödinger equation via intersection of eigenvalues. In Proceedings of the 44th IEEE Conference on Decision and Control, pages 1080-1085, 2005.

[3] A. Agrachev, Y. Baryshnikov, and A. Sarychev. Ensemble controllability by Lie algebraic methods. ESAIM Control Optim. Calc. Var., 22(4):921-938, 2016.

[4] C. Altafini and F. Ticozzi. Modeling and control of quantum systems: an introduction. IEEE Trans. Automat. Control, 57(8):1898-1917, 2012.

[5] K. Beauchard, J.-M. Coron, and P. Rouchon. Controllability issues for continuous-spectrum systems and ensemble controllability of Bloch equations. Comm. Math. Phys., 296(2):525$557,2010$.

[6] M. Belhadj, J. Salomon, and G. Turinici. Ensemble controllability and discrimination of perturbed bilinear control systems on connected, simple, compact Lie groups. Eur. J. Control, 22:23-29, 2015.

[7] A. M. Bloch, R. W. Brockett, and C. Rangan. Finite controllability of infinite-dimensional quantum systems. IEEE Transactions on Automatic Control, 55(8):1797-1805, 2010.

[8] U. Boscain, M. Caponigro, T. Chambrion, and M. Sigalotti. A weak spectral condition for the controllability of the bilinear Schrödinger equation with application to the control of a rotating planar molecule. Comm. Math. Phys., 311(2):423-455, 2012.

[9] U. Boscain, F. Chittaro, P. Mason, and M. Sigalotti. Adiabatic control of the Schroedinger equation via conical intersections of the eigenvalues. IEEE Trans. Automat. Control, 57(8):1970-1983, 2012.

[10] U. Boscain, J.-P. Gauthier, F. Rossi, and M. Sigalotti. Approximate controllability, exact controllability, and conical eigenvalue intersections for quantum mechanical systems. Comm. Math. Phys., 333(3):1225-1239, 2015.

[11] U. Boscain, P. Mason, G. Panati, and M. Sigalotti. On the control of spin-boson systems. J. Math. Phys., 56(9):092101, 15, 2015.

[12] U. Boscain and M. Sigalotti. Ensemble control of parameter-dependent quantum systems by adiabatic evolution. In Proceedings of the 56th IEEE Conference on Decision and Control, 2017.

[13] C. Brif, R. Chakrabarti, and H. Rabitz. Control of quantum phenomena: past, present and future. New Journal of Physics, 12(7):075008, 2010.

[14] S. Chelkowski, A. D. Bandrauk, and P. B. Corkum. Efficient molecular dissociation by a chirped ultrashort infrared laser pulse. Phys. Rev. Lett., 65:2355-2358, 1990.

[15] C. Chen, D. Dong, R. Long, I. R. Petersen, and H. A. Rabitz. Sampling-based learning control of inhomogeneous quantum ensembles. Physical Review A, 89(2):023402, 2014.

[16] F. Chittaro and P. Mason. Approximate controllability by adiabatic methods of the Schrödinger equation with nonlinear Hamiltonian. Proceedings of the IEEE 54th Annual Conference on Decision and Control (CDC), Osaka, Japan, pages 7771-7776, 2015. 
[17] F. C. Chittaro and J.-P. Gauthier. Asymptotic ensemble stabilizability of the Bloch equation. Systems Control Lett., 113:36-44, 2018.

[18] F. C. Chittaro and P. Mason. Approximate controllability via adiabatic techniques for the three-inputs controlled Schrödinger equation. SIAM J. Control Optim., 55(6):4202-4226, 2017.

[19] D. D'Alessandro. Introduction to quantum control and dynamics. Applied Mathematics and Nonlinear Science Series. Boca Raton, FL: Chapman, Hall/CRC., 2008.

[20] G. Dirr. Ensemble controllability of bilinear systems. Oberwolfach Reports, 9(1):674-676, 2012.

[21] D. Dong and I. R. Petersen. Quantum control theory and applications: a survey. IET Control Theory \&S Applications, 4(12):2651-2671, 2010.

[22] U. Gaubatz, P. Rudecki, S. Schiemann, and K. Bergmann. Population transfer between molecular vibrational levels by stimulated Raman scattering with partially overlapping laser fields. a new concept and experimental results. The Journal of Chemical Physics, 92(9):53635376, 1990.

[23] J.-P. Gauthier and V. Zakalyukin. On the codimension one motion planning problem. J. Dyn. Control Syst., 11(1):73-89, 2005.

[24] S. J. Glaser, U. Boscain, T. Calarco, C. P. Koch, W. Köckenberger, R. Kosloff, I. Kuprov, B. Luy, S. Schirmer, T. Schulte-Herbrüggen, D. Sugny, and F. K. Wilhelm. Training Schrödinger's cat: quantum optimal control. Strategic report on current status, visions and goals for research in Europe. European Physical Journal D, 69:279, 2015.

[25] M. Goresky and R. MacPherson. Stratified Morse theory, volume 14 of Ergebnisse der Mathematik und ihrer Grenzgebiete (3) [Results in Mathematics and Related Areas (3)]. SpringerVerlag, Berlin, 1988.

[26] U. Helmke and M. Schönlein. Uniform ensemble controllability for one-parameter families of time-invariant linear systems. Systems Control Lett., 71:69-77, 2014.

[27] S. Jansen, M.-B. Ruskai, and R. Seiler. Bounds for the adiabatic approximation with applications to quantum computation. Journal of Mathematical Physics, 48(10):102111, 2007.

[28] T. Kato. Perturbation theory for linear operators. Classics in Mathematics. Springer-Verlag, Berlin, 1995. Reprint of the 1980 edition.

[29] M. Keyl, R. Zeier, and T. Schulte-Herbrueggen. Controlling Several Atoms in a Cavity. New J. Phys., 16:065010, 2014.

[30] C. K. Law and J. H. Eberly. Arbitrary control of a quantum electro-magnetic field. Phys. Rev. Lett., 76(7):1055-1058, 1996.

[31] Z. Leghtas, A. Sarlette, and P. Rouchon. Adiabatic passage and ensemble control of quantum systems. Journal of Physics B, 44(15), 2011.

[32] J.-S. Li. Ensemble control of finite-dimensional time-varying linear systems. IEEE Trans. Automat. Control, 56(2):345-357, 2011.

[33] J.-S. Li and N. Khaneja. Control of inhomogeneous quantum ensembles. Phys. Rev. A, 73:030302, 2006.

[34] J.-S. Li and N. Khaneja. Ensemble control of Bloch equations. IEEE Trans. Automat. Control, 54(3):528-536, 2009.

[35] J.-S. Li and J. Qi. Ensemble control of time-invariant linear systems with linear parameter variation. IEEE Trans. Automat. Control, 61(10):2808-2820, 2016.

[36] E. Paduro and M. Sigalotti. Approximate controllability of the two trapped ions system. Quantum Information Processing, 14:2397-2418, 2015.

[37] M. Reed and B. Simon. Methods of modern mathematical physics. IV. Analysis of operators. Academic Press [Harcourt Brace Jovanovich, Publishers], New York-London, 1978.

[38] F. Rellich. Perturbation theory of eigenvalue problems. Assisted by J. Berkowitz. With a preface by Jacob T. Schwartz. Gordon and Breach Science Publishers, New York, 1969.

[39] E. A. Shapiro, V. Milner, and M. Shapiro. Complete transfer of populations from a single state to a preselected superposition of states using piecewise adiabatic passage: Theory. Physical Review A, 79(2):023422, 2009.

[40] B. W. Shore. The theory of coherent atomic excitation. Vol. 1. A Wiley-Interscience Publication. John Wiley \& Sons, Inc., New York, 1990. Simple atoms and fields.

[41] B. W. Shore. The theory of coherent atomic excitation. Vol. 2. A Wiley-Interscience Publication. John Wiley \& Sons, Inc., New York, 1990. Multilevel atoms and incoherence.

[42] T. E. Skinner, T. O. Reiss, B. Luy, N. Khaneja, and S. J. Glaser. Application of optimal control theory to the design of broadband excitation pulses for high-resolution NMR. Journal of Magnetic Resonance, 163(1):8-15, 2003.

[43] S. Teufel. Adiabatic perturbation theory in quantum dynamics, volume 1821 of Lecture Notes in Mathematics. Springer-Verlag, Berlin, 2003.

[44] J. von Neumann and E. Wigner. Überdas Verhalten von Eigenwerten bei Adiabatischen 
Prozessen. Z. Phys., 30:467-470, 1929.

[45] H. Yuan and S. Lloyd. Controllability of the coupled spin- $\frac{1}{2}$ harmonic oscillator system. Physical Review A, 75:052331, 2007. 